\title{
Time Series Copulas for Heteroskedastic Data
}

\author{
Rubén Loaiza-Maya, Michael S. Smith and Worapree Maneesoonthorn \\ First Version March 2016 \\ $=$ \\ This Version July 2017
}

Rubén Loaiza-Maya is a PhD student, Michael Smith is Chair of Management (Econometrics) and Worapree Maneesoonthorn is Assistant Professor of Statistics and Econometrics, all at Melbourne Business School, University of Melbourne. Correspondence should be directed to Michael Smith at mike.smith@mbs.edu. We thank the editor Prof. Andrew Patton and two anonymous referees for corrections and constructive comments that have improved the paper. We would also like to thank participants at the 2016 Melbourne Bayesian Econometrics Workshop, and the 10th International Conference on Computational and Financial Econometrics in Seville, for useful feedback. This work was partially supported by Australian Research Council Future Fellowship FT110100729.

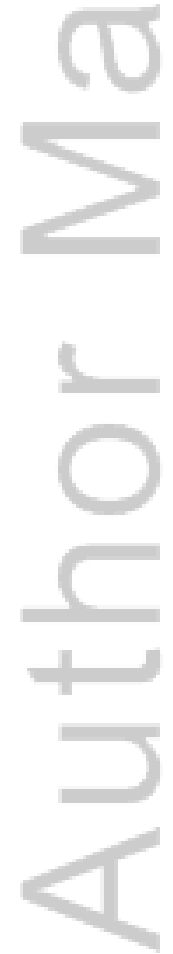

This is the author manuscript accepted for publication and has undergone full peer review but has not been through the copyediting, typesetting, pagination and proofreading process, which may lead to differences between this version and the Version of Record. Please cite this article as doi: $10.1002 /$ jae. 2610 


\title{
Time Series Copulas for Heteroskedastic Data
}

\begin{abstract}
We propose parametric copulas that capture serial dependence in stationary heteroskedastic time series. We suggest copulas for first order Markov series, and then extend them to higher orders and multivariate series. We derive the copula of a volatility proxy, based on which we propose new measures of volatility dependence, including co-movement and spillover in multivariate series. In general, these depend upon the marginal distributions of the series. Using exchange rate returns, we show that the resulting copula models can capture their marginal distributions more accurately than univariate and multivariate GARCH models, and produce more accurate value at risk forecasts.
\end{abstract}

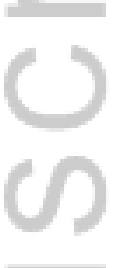

Key Words: Foreign Exchange Returns; Mixture Copula; Multivariate Time Series; Vines; Volatility Spillover and Co-movement; Value at Risk Forecasting 


\section{Introduction}

While parametric copulas are widely used to model cross-sectional dependence in multivariate time series (Patton, 2012), they are also increasingly employed to capture serial dependence in time series. We refer to the latter as 'time series copulas'. Darsow et al. (1992) and Ibragimov (2009) provide characterizations of time series copulas for univariate Markov processes, while Joe (1997), Lambert and Vandenhende (2002), Chen and Fan (2006), Domma et al. (2009), Chen et al. (2009), Beare (2010) and Beare (2012) use Archimedean or elliptical copulas to capture serial dependence in this case. Smith et al. (2010) use vine copulas to capture serial dependence in non-stationary longitudinal data. For multivariate time series, Biller and Nelson (2003), Rémillard et al. (2012), Smith (2015) and Beare and Seo (2015) use elliptical, Archimedean or vine copulas to account for serial dependence. However, all these copulas can prove inadequate when a time series exhibits heteroskedasticity. For example, Smith and Vahey (2016) fit a Gaussian time series copula model to heteroskedastic multivariate time series data, but note that it has limited ability to represent serial dependence in the conditional variance. To address this problem, we propose closed form parametric copulas to capture serial dependence in heteroskedastic series. Using these, we construct new time series models for heteroskedastic continuous-valued data that also allow for flexible marginssomething that is difficult to achieve using existing nonlinear time series models.

Heteroskedasticity is a key feature of many financial and economic time series. In the multivariate case, many authors employ existing univariate time series models for each series, along with a copula to account for conditional cross-sectional dependence only. Additional flexibility is introduced with a dynamic specification of the time-varying copula parameters (Patton, 2006, Almeida and Czado, 2012, Hafner and Manner, 2012, Oh and Patton, 2013, De Lira Salvatierra and Patton, 2015, Creal and Tsay, 2015). As an alternative, Smith and Maneesoonthorn (2016) consider extracting implicit or 'inversion' copulas from univariate state space models numerically, including for heteroskedastic models. However, as far as we are aware, closed form copulas that can adequately account for serial dependence of heteroskedastic data have yet to be investigated. To do so, we compute empirically the bivariate copula density of first order serial dependence for two popular stationary heteroskedastic time series models. Both densities have an unusual cross shape, with mass concentrated at all four corners of the unit square. The level of concentration increases with the level of volatility persistence. We approximate these copulas using a mixture of bivariate copulas. When combined with a flexible marginal distribution, the resulting copula model can be employed to model a wide range of heteroskedastic time series with Markov order one. To illustrate, Figure 1 plots four simulated time series. Each series has the same first order serial dependence structure, given by a mixture of bivariate copulas that we discuss later in Section 2.1, but with four different margins: (a) Gaussian, (b) t, (c) Beta and (d) Log-normal. Each series exhibits common features of heteroskedastic data, such as volatility clustering, even when the margin is bounded or skewed.

We extend our copula to higher Markov orders $p>1$ using a drawable vine (or 'D-vine') copula. These are compositions of bivariate copula components called 'pair-copulas' (Aas et al., 2009). Vine copulas can be difficult to use in high dimensions because the number of pair-copulas and possible decompositions can be large. However, even though the dimension is high in the time series case, there is only one D-vine decomposition, which is parsimonious when the series is Markov and stationary. Beare and Seo (2015), Brechmann and Czado (2015) and Smith (2015) all show that parsimonious vine copulas can also be used to capture cross-sectional and serial dependence jointly in multivariate time series. We follow these authors and employ a D-vine copula for multivariate heteroskedastic 
data, but with pair-copulas given by our proposed bivariate mixture copula.

Existing popular dependence measures computed from the time series copula are poor measures of volatility dependence. A major contribution of the paper is that we derive new alternative measures. To obtain these we consider a volatility proxy that is a transformation of the series, and derive the bivariate copula of the proxy at any two points in time. We label this a 'volatility copula', and show that it is invariant to specific choice of transformation, given some broad properties that are consistent with a volatility proxy. Then pairwise dependence measures - such as Spearman's rho or Kendall's tau-computed from this volatility copula can be used to measure volatility dependence. These pairwise measures can also be computed in the multivariate time series case, forming new measures of volatility persistence, co-movement and spillover. The proposed measures of volatility dependence are general for two reasons. First, they are not based on a specific structural assumption for the conditional variance of the series, as is the case with most existing models such as the BEKK (Engle and Kroner, 1995) and DCC (Engle, 2002) models. Second, they can be computed for any time series model, so that the degree and type of volatility dependence of different models can be compared. To the best of our knowledge, ours is the first study to propose measuring volatility dependence from the copula perspective.

The density of our bivariate mixture copula is available in closed form, so that the model likelihoods are also. We outline parallel algorithms to compute these efficiently for the vine copulas. These are extensions of that originally proposed by Aas et al. (2009) to exploit the parsimonious structure of the vine copulas in the time series case. Maximum likelihood estimation (MLE) is straightforward for univariate series with low Markov orders, but for larger vines we follow Min and Czado (2010), Smith et al. (2010) and Smith (2015), and compute the posterior distribution using Markov chain Monte Carlo (MCMC) methods.

To illustrate the advantages of our new methodology we apply it to daily foreign exchange returns. These exhibit strong heteroskedasticity, but have marginal distributions that are typically asymmetric and fat-tailed (Boothe and Glassman, 1987). Capturing such nuanced margins is difficult using most existing time series models, but is easy in the copula framework. We first employ a univariate time series copula model for USD/AUD returns, and compare it to GARCH alternatives. We then extend the study to also include USD/EUR and USD/JPY returns in a trivariate time series copula model, and compare it to multivariate GARCH alternatives. The GARCH models are shown to have inaccurate margins, whereas our copula models employ more accurate nonparametric estimates. We compute our new metrics of volatility dependence for all models, and find the copula models capture positive volatility persistence similar to the benchmark models. However, in the multivariate case the copula model also captures both positive volatility co-movements and spillovers, whereas those from the multivariate GARCH models are restricted. In a validation study we find that the one day ahead Value-at-Risk (VaR) forecasts from the copula models are more accurate than those from the GARCH models - both in the univariate and multivariate cases. A small simulation study also shows that our copulas are more robust to model misspecification than GARCH equivalents.

The paper is organized as follows. In Section 2 we outline the proposed copula model. We derive the volatility copula, and show how to use it to measure volatility persistence. The section concludes with the analysis of USD/AUD exchange rate returns, validation and simulation studies. Section 3 extends the methodology to multivariate time series, and is employed to model jointly the three exchange rate returns series, while Section 4 concludes. 


\section{Heteroskedastic Time Series}

\subsection{Copulas of Serial Dependence}

Following Sklar (1959), the joint distribution function of $T$ observations $\boldsymbol{y}=\left(y_{1}, \ldots, y_{T}\right)$ on a time series can be written as

$$
F(\boldsymbol{y})=C(\boldsymbol{u}) .
$$

Here, $\boldsymbol{u}=\left(u_{1}, \ldots, u_{T}\right), u_{t}=F_{t}\left(y_{t}\right), F_{t}$ is the marginal distribution function of $y_{t}$, and $C$ is a $T$ dimensional copula function that captures the serial dependence in the time series. Copula functions are usually selected from a range of parametric copulas when modeling cross-sectional dependence; see, for example, Nelsen (2006) and Joe (2014). However, only limited consideration has been given to an appropriate choice of $C$ when modeling serial dependence. We consider this here when the time series is heteroskedastic.

If the time series is continuous, then the density of $\boldsymbol{y}$ is

$$
f(\boldsymbol{y})=c(\boldsymbol{u}) \prod_{t=1}^{T} f_{t}\left(y_{t}\right)
$$

where $f_{t}\left(y_{t}\right)=\frac{\partial}{\partial y_{t}} F_{t}\left(y_{t}\right)$, and $c(\boldsymbol{u})=\frac{\partial^{T}}{\partial u_{1}, \ldots, \partial u_{T}} C(\boldsymbol{u})$ is the copula density. Note that throughout this paper, copula functions are denoted with upper case $C$, and copula densities with lower case $c$. If the time series $\left\{y_{t}\right\}$ is strongly stationary (Brockwell and Davis, 1991) and Markov order one, then it is straightforward to show that the series $\left\{u_{t}\right\}$ is also (Smith, 2015). In this case, the copula density can be greatly simplified as

$$
c(\boldsymbol{u})=\prod_{t=2}^{T} f\left(u_{t} \mid u_{t-1}\right)=\prod_{t=2}^{T} c_{2}\left(u_{t-1}, u_{t}\right)
$$

so that the serial dependence is captured by a single bivariate copula with density $c_{2}$. (Note that we choose this notation for the copula to be consistent with that used later for the vine copula at Equation (5).)

To explore the shape of $c_{2}$ for conditionally heteroskedastic time series, we consider two popular Markovian processes. The first is the $\mathrm{ARCH}(1)$ model, where $y_{t}=\epsilon_{t} \sigma_{t}, \sigma_{t}^{2}=\alpha_{0}+\alpha_{1} y_{t-1}^{2}$, and $\epsilon_{t} \sim N(0,1)$. The second is the first order stochastic volatility model $(\mathrm{SV}(1))$, where $y_{t}=\epsilon_{t} \exp \left(\frac{h_{t}}{2}\right)$, $\left(h_{t}-\bar{h}\right)=\phi_{1}\left(h_{t-1}-\bar{h}\right)+\eta_{t}$, and $\eta_{t} \sim N\left(0, \sigma^{2}\right)$. Figure 2(a,b) displays empirical copula density estimates of $c_{2}$ for the $\operatorname{ARCH}(1)$ model with medium $\left(\alpha_{0}=0.01, \alpha_{1}=0.5\right)$, and high $\left(\alpha_{0}=0.01, \alpha_{1}=\right.$ 0.9 ) persistence. These are obtained by simulating $T=50,000$ observations from each process, estimating the time-invariant margins of $y_{t}$ using a locally adaptive kernel density estimator, from which copula data are computed. Each panel then displays a bivariate histogram of the copula data and their values lagged one period. Both time series show positive and equally-valued tail dependence in all four quadrants, along with a shallow mode around $(0.5,0.5)$. Higher persistence results in higher tail dependence, along with a more pronounced central mode in $c_{2}$. Similar features can also be seen in Figure 2(c,d), which displays the empirical copula density estimates of $c_{2}$ for the SV(1) model with medium $\left(\bar{h}=0.8, \sigma^{2}=2.5, \phi_{1}=0.5\right)$ and high $\left(\bar{h}=0.8, \sigma^{2}=2, \phi_{1}=0.9\right)$ persistence. We note that despite the strong serial dependence in these series, Kendall's tau and Spearman's rho- the two most commonly employed measures of dependence- of $c_{2}$ can be shown to be exactly zero. 
Few bivariate copulas can replicate the features found in Figure $2(\mathrm{a}-\mathrm{d})$. One copula that can do so is the t copula (Demarta and McNeil, 2005) with a correlation parameter and degrees of freedom both close to zero 1 However, the t copula has symmetric tail dependence and becomes numerically unstable as the degrees of freedom approaches zero. A more flexible alternative is to employ a mixture of rotated copulas, which are increasingly popular in other contexts; see Fortin and Kuzmics (2002), Smith (2015) and Oh and Patton (2016) among others. Let $C^{a}, C^{b}$ and $c^{a}, c^{b}$ be copula functions and densities of two parametric bivariate copulas that both have non-negative Kendall's tau. (We label these copulas using superscripts to avoid confusion with pair-copulas indices employed later.) Then, we use a mixture of $c^{a}$ and a 90 degree rotation of $c^{b}$, with density

$$
c^{M I X}(u, v ; \gamma)=w c^{a}\left(u, v ; \boldsymbol{\gamma}^{a}\right)+(1-w) c^{b}\left(1-u, v ; \gamma^{b}\right), 0 \leq w \leq 1
$$

and parameters $\gamma=\left\{w, \gamma^{a}, \gamma^{b}\right\}$, for $c_{2}$ in Equation (3). For example, $t$ copulas with positive correlation parameters can be used for $c^{a}$ and $c^{b}$, so that $\gamma^{a}=\left(\zeta^{a}, \nu^{a}\right), \gamma^{b}=\left(\zeta^{b}, \nu^{b}\right)$, with $\zeta^{a}>0$ and $\zeta^{b}>0$ the correlation parameters, and $\nu^{a}$ and $\nu^{b}$ the degrees of freedom. The four series in Figure 1 were simulated using such a copula for $c_{2}$ with $w=0.5, \zeta^{a}=\zeta^{b}=0.9, \nu^{a}=\nu^{b}=3$. Each element $u_{t}$ was transformed to $y_{t}$ using the quantile functions of the four marginal distributions.

In our empirical work, for the mixture components $c^{a}$ and $c^{b}$ we use either t copulas or or 'convex Gumbels', where the latter is defined as follows. Let $c^{G}(u, v ; \tau)$ be the density of a Gumbel copula parameterized (uniquely) in terms of its Kendall tau $\tau \geq 0$. Then the convex Gumbel has a density $c^{c G}$ equal to the convex combination of that of the Gumbel and it's rotation 180 degrees (ie. the survival copula), so that

$$
c^{c G}(u, v ; \tau, \delta)=\delta c^{G}(u, v ; \tau)+(1-\delta) c^{G}(1-u, 1-v ; \tau),
$$

with $0 \leq \delta \leq 1$. This copula was suggested by Junker and May (2005). When employed in Equation (44), it gives a five parameter bivariate copula with $\gamma^{a}=\left(\delta^{a}, \tau^{a}\right), \gamma^{b}=\left(\delta^{b}, \tau^{b}\right)$, and a density $c^{M I X}$ that is equal to a mixture of all four 90 degree rotations of the Gumbel, similar to the jointly symmetric copula of $\mathrm{Oh}$ and Patton (2016) in the bivariate case. Table 1 gives the copula functions $C^{M I X}, C^{c G}$ for both the mixture and convex Gumbel copulas.

To show that $C^{M I X}$ can reproduce the features exhibited by the empirical copulas in Figure 2(ad), we fit it (with $t$ copulas for $c^{a}$ and $c^{b}$ ) to the same four copula datasets. The parameters $\gamma$ are estimated by maximizing the copula density at Equation (3), which is the likelihood conditional on the copula data (the point estimates are reported in the Online Appendix). Figure 2 (e-h) plots $c_{2}$ for the four estimated copulas, and in each case the mixture copula reproduces the shape of the corresponding empirical copula well. To show the mixture copulas also replicate the quantile dependence, we compute the quantile dependence coefficients $\lambda_{\text {low }}(\alpha)=P\left(u_{t}<\alpha \mid u_{t-1}<\alpha\right)$ and $\lambda_{\text {up }}(\alpha)=P\left(u_{t}>\alpha \mid u_{t-1}>\alpha\right)$, for both the fitted mixture copulas and the empirical copulas. Figure 3 plots these coefficients against $\alpha$, where $\lambda_{\text {low }}(\alpha)$ is plotted for $0<\alpha<0.5$, and $\lambda_{\text {up }}(\alpha)$ for $0.5<\alpha<1$. The coefficients of the mixture and empirical copulas are very close. Figure ?? in the Online Appendix shows that the same is true for the quantile dependence coefficients in the off-diagonal quadrants.

For Markov processes of order $p>1$, we follow Smith et al. (2010) and use a drawable vine (or 'Dvine'). A vine copula density is equal to the product of the densities of a sequence of bivariate copula

\footnotetext{
${ }^{1}$ We thank a referee who pointed this out to us, and that the bivariate t copula is well-defined when the degrees of freedom $0<\nu<2$.
} 
components, called 'pair-copulas' (Aas et al., 2009). In a general D-vine there are $T(T-1) / 2$ of these, although in our stationary time series case there are only $p$ unique pair-copulas and a single ordering of the variables (i.e. the time order). To define the D-vine, for $s<t$ denote $u_{t \mid s}=F\left(u_{t} \mid u_{s}, \ldots, u_{t-1}\right)$, $u_{s \mid t}=F\left(u_{s} \mid u_{s+1}, \ldots, u_{t}\right)$ and $u_{t \mid t}=u_{t}=F_{t}\left(y_{t}\right)$. Then, as shown in Appendix $\mathrm{A}$, the D-vine copula density is

$$
\begin{aligned}
c^{D V}(\boldsymbol{u}) & =\prod_{t=2}^{T} f\left(u_{t} \mid u_{\max (1, t-p)}, \ldots, u_{t-1}\right) \\
& =\prod_{t=2}^{T} \prod_{k=1}^{\min (t-1, p)} c_{k+1}\left(u_{t-k \mid t-1}, u_{t \mid t-k+1} ; \gamma_{k+1}\right)
\end{aligned}
$$

When $p=1, c^{D V}$ is equal to the density at Equation (33). Each pair-copula density $c_{k+1}$ has a parameter vector $\gamma_{k+1}$, which we denote explicitly. When $k>1, c_{k+1}$ captures dependence between $y_{t-k}$ and $y_{t}$, conditional on the intervening observations $\left(y_{t-k+1}, \ldots, y_{t-1}\right)$. When the series is strongly stationary, the bivariate distribution of $y_{t-k}, y_{t} \mid y_{t-k+1}, \ldots, y_{t-1}$ does not vary with $t$, so that neither does the pair-copula density $c_{k+1}$ nor the parameters $\gamma_{k+1}$. We use mixture copulas with densities given at Equation (4) for each of the pair-copula components $c_{2}, \ldots, c_{p+1}$. Therefore, each pair-copula $c_{k+1}$ has parameter vector $\gamma_{k+1}=\left\{w_{k+1}, \gamma_{k+1}^{a}, \gamma_{k+1}^{b}\right\}$, and the vine copula density $c^{D V}$ has parameters $\gamma=\left\{\gamma_{2}, \ldots, \gamma_{p+1}\right\}$. Last, the pair-copula arguments $u_{t \mid s}, u_{s \mid t}$ are computed from $\boldsymbol{u}$ using the efficient algorithm in Appendix C.1.

We show how the vine copula can replicate the serial dependence characteristics of three $\mathrm{ARCH}(3)$ models in the Online Appendix.

\subsection{Measuring Persistence in Volatility}

We measure serial dependence in the series values using the bivariate marginal copulas

$$
\bar{c}\left(u_{t-k}, u_{t}\right)=\int c^{D V}(\boldsymbol{u}) d u_{j \notin\{t-k, t\}},
$$

for $k \geq 1$. When $k=1$, the marginal copula is simply the pair-copula $c_{2}\left(u_{t-1}, u_{t} ; \gamma_{2}\right)$. When $k>1$, the marginal copulas are unavailable in closed form, but can be computed via simulation from the D-vine; see Smith et al. (2010) for details on how to simulate from a vine copula. However, popular pairwise dependence measures computed from these marginal copulas do not measure volatility persistence. For example, for the ARCH and SV processes above, both Spearman's rho and Kendall's tau of $c_{2}$ are exactly zero.

We therefore propose new measures of volatility persistence. These are computed from the bivariate copulas of $\left(v_{t-k}, v_{t}\right)$, for $k \geq 1$, where $v_{t}=V\left(y_{t}-E\left(y_{t}\right)\right)$ is a transformation of the mean-corrected time series values. The smooth transformation $V: \mathbb{R} \rightarrow \mathbb{R}^{+}$is defined so that:

(i) $V(a)=V(-a) \geq 0$, and $V(0)=0$ (symmetry around zero), and

(ii) $\frac{d}{d a} V(a)>0$ if $a>0$, and $\frac{d}{d a} V(a)<0$ if $a<0$.

Examples include $V(a)=|a|$ and $V(a)=a^{2}$, and we label the copula of $\left(v_{t-k}, v_{t}\right)$ a 'volatility copula'. Measures of dependence computed from this volatility copula are pairwise measures of volatility persistence in the time series at lag $k$. The copula functions and densities of these transformed time series values are given by the following theorem. 
Theorem 1 For $s<t$, let $y_{s}, y_{t}$ be time series observations with marginal distribution functions $F_{s}, F_{t}$, marginal means $\mu_{s}, \mu_{t}$, bivariate marginal copula function $\bar{C}$ and density $\bar{c}$. Then the copula function of the transformed values $v_{s}=V\left(y_{s}-\mu_{s}\right), v_{t}=V\left(y_{t}-\mu_{t}\right)$ is

$$
C_{V}\left(\tilde{u}_{s}, \tilde{u}_{t}\right)=\sum_{i=1}^{2} \sum_{j=1}^{2}(-1)^{i}(-1)^{j} \bar{C}\left(F_{s}\left(\mu_{s}+(-1)^{i} G\left(F_{V_{s}}^{-1}\left(\tilde{u}_{s}\right)\right)\right), F_{t}\left(\mu_{t}+(-1)^{j} G\left(F_{V_{t}}^{-1}\left(\tilde{u}_{t}\right)\right)\right)\right)
$$

with corresponding density

$$
c_{V}\left(\tilde{u}_{s}, \tilde{u}_{t}\right)=\frac{\sum_{i=1}^{2} \sum_{j=1}^{2} f\left(\mu_{s}+(-1)^{i} G\left(F_{V_{s}}^{-1}\left(\tilde{u}_{s}\right)\right), \mu_{t}+(-1)^{j} G\left(F_{V_{t}}^{-1}\left(\tilde{u}_{t}\right)\right)\right) G^{\prime}\left(F_{V_{s}}^{-1}\left(\tilde{u}_{s}\right)\right) G^{\prime}\left(F_{V_{t}}^{-1}\left(\tilde{u}_{t}\right)\right)}{f_{V_{s}}\left(F_{V_{s}}^{-1}\left(\tilde{u}_{s}\right)\right) f_{V_{t}}\left(F_{V_{t}}^{-1}\left(\tilde{u}_{t}\right)\right)}
$$

where

$$
\begin{aligned}
F_{V_{j}}\left(v_{j}\right) & =F_{j}\left(G\left(v_{j}\right)+\mu_{j}\right)-F_{j}\left(-G\left(v_{j}\right)+\mu_{j}\right), \\
f_{V_{j}}\left(v_{j}\right) & =\left(f_{j}\left(G\left(v_{j}\right)+\mu_{j}\right)+f_{j}\left(-G\left(v_{j}\right)+\mu_{j}\right)\right) G^{\prime}\left(v_{j}\right),
\end{aligned}
$$

are the marginal distribution and density functions of $v_{j}$, for $j \in\{s, t\}, \tilde{u}_{j}=F_{V_{j}}\left(V\left(y_{j}-\mu_{j}\right)\right)$, and $G$ is a differentiable function such that $G(V(a))=|a|$ for any $a \in \mathbb{R}$.

Proof: See Appendix B.1.

Note that in Theorem 1 above we do not index $\bar{C}, C_{V}, \bar{c}$ and $c_{V}$ by $s, t$ to aid readability.

We make a number of observations about the expressions for $C_{V}$ and $c_{V}$ in Theorem 1. First, they do not vary with specific choice of transformation $V$. Consequently, measures of dependence computed from this copula are also invariant with respect to $V$, and in this way are general measures of volatility persistence. Second, they can be computed analytically, except for the inversion of $F_{V_{j}}$, which is numerical. Third, they apply equally to stationary or non-stationary time series $\left\{y_{t}\right\}$. However, in the former case, $C_{V}$ and $c_{V}$ can be further simplified because the margin is time invariant, so that $F_{s}=F_{t}$ for all $s, t$. Last, the expressions are not only a function of the marginal copula of $\left(y_{s}, y_{t}\right)$, but also of the margins $F_{s}, F_{t}$. The implication for applied modeling is that the choice of copula at Equation (1) does not solely determine the form and degree of persistence in the volatility of the series $\left\{y_{t}\right\}$.

When both $F_{s}$ and $F_{t}$ are symmetric, the expressions for $C_{V}$ and $c_{V}$ are simplified as below.

Lemma 1 If $F_{s}$ and $F_{t}$ are both symmetric, then

$$
\begin{gathered}
C_{V}\left(\tilde{u}_{s}, \tilde{u}_{t}\right)=\sum_{i=1}^{2} \sum_{j=1}^{2}(-1)^{i}(-1)^{j} \bar{C}\left(\frac{1+(-1)^{i} \tilde{u}_{s}}{2}, \frac{1+(-1)^{j} \tilde{u}_{t}}{2}\right), \text { and } \\
c_{V}\left(\tilde{u}_{s}, \tilde{u}_{t}\right)=\frac{1}{4} \sum_{i=1}^{2} \sum_{j=1}^{2} \bar{c}\left(\frac{1+(-1)^{i} \tilde{u}_{s}}{2}, \frac{1+(-1)^{j} \tilde{u}_{t}}{2}\right) .
\end{gathered}
$$

Proof: See Appendix B.2

In Lemma 1, the expressions for $C_{V}$ and $c_{V}$ do not involve $F_{s}$ or $F_{t}$ so that, in this special case only, the persistence in the volatility of the series is unaffected by the choice of marginal distributions.

When $s=t-k$, measures of dependence computed from $C_{V}$ are persistence metrics for the volatility at lag $k \geq 1$. For example, Spearman's rho is

$$
\rho_{t-k, t}^{v}=12 \iint C_{V}\left(\tilde{u}_{t-k}, \tilde{u}_{t}\right) d \tilde{u}_{t} d \tilde{u}_{t-k}-3=12 E\left(\tilde{u}_{t-k} \tilde{u}_{t}\right)-3 .
$$


For the D-vine, when $k=1$ the marginal copula for $\left(y_{t-1}, y_{t}\right)$ is the pair-copula with density $c_{2}\left(u_{t-1}, u_{t} ; \gamma_{2}\right)$. From this, $C_{V}$ can be computed using Theorem 1, and $\rho_{t-1, t}^{v}$ at Equation (7) evaluated by bivariate numerical integration. However, when $k>1$, the marginal copula for $\left(y_{t-k}, y_{t}\right)$ is unavailable in closed form, and $\rho_{t-k, t}^{v}$ needs to be evaluated via Monte Carlo simulation. We note that because our time series model is stationary, it is straightforward to show that $\rho_{t-k, t}^{v}$ does not vary with $t$, so that we simply denote it as $\rho_{k}^{v}$. Last, other measures of dependence can be computed from $C_{V}$ similarly.

To highlight the coherence of this measure of persistence in volatility, we compute $\rho_{1}^{v}$ for the four heteroskedastic time series used to fit the copulas depicted in Figure 2. Table 2 reports these values, along with Spearman's rho between $\left(y_{t-1}, y_{t}\right)$, which we denote as $\rho_{1}^{y}$. Both metrics are computed using numerical integration for the fitted mixture copulas. For comparison, we also compute equivalent nonparametric estimates of $\rho_{1}^{v}$ and $\rho_{1}^{y}$ directly from the time series $\left\{y_{t}\right\}$ and $\left\{v_{t}\right\}$. We make three observations. First, $\rho_{1}^{y}$ is close to zero throughout, and is an inadequate measure of serial dependence for these heteroskedastic time series. Second, $\rho_{1}^{v}$ is positive throughout, and increases as the parameters $\alpha_{1}$ and $\phi_{1}$ of the $\mathrm{ARCH}(1)$ and $\mathrm{SV}(1)$ models increase. Third, the values for $\rho_{1}^{y}$ and $\rho_{1}^{v}$ computed using the fitted parametric mixture copula are similar to those computed empirically. This is further evidence that the mixture copula is an adequate parametric model of serial dependence for the heteroskedastic series. For further comparison, we also report the first order linear autocorrelations of the series, the absolute values $\left|y_{t}\right|$, and the squared values $y_{t}^{2}$. These are consistent with those from the mixture copula model, although the autocorrelations of the squared and absolute values differwhereas $\rho_{1}^{v}$ is invariant to the form of transformation $V$.

\subsection{Modeling USD/AUD Exchange Rate}

\subsubsection{First order copula model}

To illustrate the advantages of our time series copula model, we employ it to model daily returns on the USD/AUD exchange rate from 2 Jan 2001 until 7 Aug 2015, sourced from the Federal Reserve Economic Data (FRED) database. The series exhibits strong heteroskedasticity, along with an asymmetric and heavy-tailed marginal distribution. Figure 4 plots a histogram of the $T=3669$ returns, which have skew of -0.654 and kurtosis of 15.15. Also plotted are the margins of $\operatorname{GARCH}(1,1)$, EGARCH(1,1) and GARCH-t $(1,1)$ models fit to this data, computed by simulation. These models are widely used for such data (Hansen and Lunde, 2005), yet have margins that are necessarily symmetric and inaccurate. In contrast, we model the margin nonparametrically using the adaptive kernel density estimator of Shimazaki and Shinomoto (2010) - also plotted on Figure 4 - from which the copula data are computed. The use of a nonparametric time invariant margin, combined with a parametric copula, is also advocated by Chen and Fan (2006) and Chen et al. (2009) for stationary Markov series. We employ the first order time series copula at Equation (3), with the mixture copula for $c_{2}$, where $c^{a}, c^{b}$ are the densities of bivariate $\mathrm{t}$ copulas, so that there are 5 copula parameters. The resulting copula model allows for heteroskedastic serial dependence, but with a margin that is consistent with that observed empirically.

We estimate the copula parameters using both MLE and Bayesian posterior inference. For the latter, flat or uninformative proper priors are used for the copula parameters, and computation is by Markov chain Monte Carlo (MCMC), where the parameters were generated as a block using adaptive random walk Metropolis-Hastings (Roberts and Rosenthal, 2009). Table 3 reports the point estimates for both the copula parameters and serial dependence metrics. Also reported are $90 \%$ confidence intervals for the MLE, along with the $90 \%$ posterior probability intervals. We make the following 
observations. First, while the confidence and posterior intervals are wide for the parameters, those for the dependence metrics are not. This is common for copulas with multiple parameters, where a wide range of parameter values can correspond to similar copula functions and dependence values. Second, the posterior mean and MLE for the dependence metrics are almost identical. Third, return values exhibit negligible first order serial dependence $\left(\rho_{1}^{y}\right)$, but have positive first order extremal tail dependence $\left(\lambda_{\text {low }}^{y}=\lim _{\alpha \rightarrow 0} \operatorname{Pr}\left(u_{t}<\alpha \mid u_{t-1}<\alpha\right)\right)$. Last, our proposed measure of first order volatility persistence $\left(\rho_{1}^{v}\right)$ is positive, as are the corresponding quantile dependence metrics $\left(\lambda_{\text {low }}^{v}(\alpha)=\operatorname{Pr}\left(\tilde{u}_{t}<\right.\right.$ $\left.\alpha \mid \tilde{u}_{t-1}<\alpha\right)$ and $\lambda_{\text {up }}^{v}(\alpha)=\operatorname{Pr}\left(\tilde{u}_{t}>1-\alpha \mid \tilde{u}_{t-1}>1-\alpha\right)$ ) computed from the volatility copula in Theorem 1.

Finally, Figure 5 plots the fitted copula density in panel (a). For comparison, also plotted in panel (b) is the density of a first order copula model fitted to the same data, but where $c^{M I X}$ has convex Gumbel components. Both densities are very similar and have the 'cross shape' that is indicative of serial dependence in heteroskedastic series.

\subsubsection{Validation study}

Based on the USD/AUD exchange rate data, we undertake a validation study. We fit six time series copulas with D-vines of the form at Equation (5) to the copula data. They differ by order $p$ and choice of pair-copula components as follows:

Copula A1: Order $p=1$ and each pair-copula is $C^{M I X}$, where $C^{a}, C^{b}$ are t copulas.

Copula A5: Order $p=5$ and each pair-copula is $C^{M I X}$, where $C^{a}, C^{b}$ are t copulas.

Copula B1: Order $p=1$ and each pair-copula is $C^{M I X}$, where $C^{a}, C^{b}$ are convex Gumbels.

Copula B5: Order $p=5$ and each pair-copula is $C^{M I X}$, where $C^{a}, C^{b}$ are convex Gumbels.

Copula T1: Order $p=1$ and each pair-copula is a t copula.

Copula T5: Order $p=5$ and each pair-copula is a t copula.

Copula A1 is the first order model in Section 2.3, to which we add a higher order D-vine with $p=5$ and component pair-copulas of the same form. Copulas A1 and B1 are five parameter copulas, whereas Copulas A5 and B5 are parsimonious D-vines with a total of $5 \times 5=25$ parameters each. Copula T1 has only two parameters, while Copula T5 has $5 \times 2=10$ parameters. The posterior of the copula models are obtained using MCMC, where the parameters of each pair-copula were generated as a block using adaptive random walk Metropolis-Hastings, and with blocks generated in random order. Table 4 reports the deviance information criteria for each copula model. This is computed conditional on the same copula data, and is $\mathrm{DIC}_{2}$ of Celeux et al. (2006). Lower DIC 2 values are preferred, so that longer lag lengths dominate, with Copula A5 optimal by this measure.

The $\operatorname{ARCH}(1), \operatorname{GARCH}(1,1), \operatorname{GARCH}-\mathrm{t}(1,1)$ and $\operatorname{EGARCH}(1,1)$ models, estimated using MLE, are used as benchmarks. Table 4 reports the four (first order) serial dependence metrics. As expected, for all models, serial dependence in the returns $\left(\rho_{1}^{y}\right)$ is close to zero, and volatility persistence $\left(\rho_{1}^{v}\right)$ is positive. In each model, the first order $(k=1)$ volatility copula exhibits asymmetric and positive quantile dependence $\left(\lambda_{\text {up }}^{v}(0.05)>\lambda_{\text {low }}^{v}(0.05)>0\right)$, which is something that we repeatedly observe with heteroskedastic series. Interestingly, the metrics from the copula models are close to those of 
the GARCH-t $(1,1)$ model, which is widely considered the most accurate of the benchmark models for daily exchange rate returns (Baillie and Bollerslev, 2002).

To judge the quality of the fitted models, we examine one day ahead VaR coverage as follows. For each fitted model, the 1-step ahead predictive distributions $F_{t \mid t-1}\left(y_{t}\right)$ are computed for days $t=2, \ldots, T$. From these we compute $\operatorname{Va} R_{t \mid t-1}(\alpha)=F_{t \mid t-1}^{-1}(\alpha)$, for $0<\alpha<1$, along with the mean number of exceedences during the $T-1=3668$ days, defined as $\hat{\alpha}=\frac{1}{T-1} \sum_{t=2}^{T} 1\left(y_{t}<\operatorname{VaR} R_{t \mid t-1}(\alpha)\right)$. Table 5 reports $\hat{\alpha}$ for $\alpha \in\{0.01,0.05,0.1,0.9,0.95,0.99\}$, and shows that the copula models have accurate coverage. Figure 6 plots $\hat{\alpha}$ from the Copula A5 and four GARCH models against $\alpha$, for values $0.01<\alpha<0.1$ in panel (a), and $0.9<\alpha<0.99$ in panel (b). Deviations from the black 45 degree line indicate inaccurate VaR coverage, and it can be seen that the copula model dominates the GARCH models in both tails- particularly those with Gaussian innovations. We note that the predictive distributions of the GARCH models are necessarily Gaussian or t, whereas those from the copula models are not.

Last, in Table 5 we also report the results of the Christoffersen (1998) test of correct conditional coverage for all quantiles and models. This test assesses jointly whether the empirical coverage equates to the corresponding theoretical value and whether the exceedences are serially independent. Models that produce forecasts that fail to reject the conditional coverage test are deemed to perform well in predicting VaR. The test results suggest all copula models dominate the GARCH benchmarks, except for Copula T1 which is the least flexible.

\subsection{Simulation Study}

To illustrate the robustness of the time series copula to model misspecification we undertake a small simulation study based on the USD/AUD exchange rate data. One hundred datasets, each of length $T=3669$, were simulated from the fitted $\mathrm{ARCH}(1)$, Copula B1, GARCH(1,1) and Copula B5 models in Section 2.3.2 above. For each model and dataset we fit both the correct and one incorrect model (listed in Table 6), giving eight fitted models in total.

To measure the accuracy of the estimated volatility dependence, we compute $\rho_{1}^{v}, \ldots, \rho_{5}^{v}$ from the fitted models. These coefficients are computed by simulating series of length 1 million from the models, and then computing the sample Spearman's rho of $V\left(y_{t}\right)$ and $V\left(y_{t-k}\right)$ for $k=1, \ldots, 5$. We repeat this for all 100 datasets and compute the root mean squared error (RMSE) for each coefficient and fitted model. Here, the true value of $\rho_{k}^{v}$ can be computed accurately via simulation from the true model as well. Table 6 reports the ratio of the RMSE values of the misspecified models, relative to that obtained by fitting the correct model. Greater relative RMSE values indicate that the fitted misspecified model does not capture the volatility serial dependence well. The results indicate that the two copula models reproduce the volatility serial dependence structure of the GARCH models well, although the converse is not true. For example, for $\rho_{1}^{v}$ the relative RMSEs of incorrectly fitting the Copula B1 and B5 models are only 1.16 and 1.04; yet the relative RMSEs are 3.41 and 3.25 when incorrectly fitting the $\mathrm{ARCH}(1)$ and $\operatorname{GARCH}(1,1)$ models. 


\section{Multivariate Heteroskedastic Time Series}

\subsection{Copula Model}

Copulas can also be used to model dependence in multivariate time series. The copula model for the $T$ observations $\boldsymbol{y}=\left(\boldsymbol{y}_{1}^{\prime}, \ldots, \boldsymbol{y}_{T}^{\prime}\right)^{\prime}$ of a vector $\boldsymbol{y}_{t}=\left(y_{1, t}, \ldots, y_{m, t}\right)^{\prime}$ of $m$ continuous values has density

$$
f(\boldsymbol{y})=c(\boldsymbol{u}) \prod_{t=1}^{T} \prod_{j=1}^{m} f_{j, t}\left(y_{j, t}\right)
$$

where $\boldsymbol{u}^{=}=\left(\boldsymbol{u}_{1}^{\prime}, \ldots, \boldsymbol{u}_{T}^{\prime}\right)^{\prime}, \boldsymbol{u}_{t}=\left(u_{1, t}, \ldots, u_{m, t}\right)^{\prime}, u_{j, t}=F_{j, t}\left(y_{j, t}\right), F_{j, t}$ is the marginal distribution function of $y_{j, t}$, and $f_{j, t}\left(y_{j, t}\right)=\frac{d}{d y_{j, t}} F_{j, t}\left(y_{j, t}\right)$. The copula density in Equation (8) is of dimension $m T$, and captures both serial and cross-sectional dependence in the series jointly. Selection of an appropriate high-dimensional copula is the main challenge in constructing the model.

Biller and Nelson (2003), Biller (2009) and Smith and Vahey (2016) all use Gaussian copulas, with parameter matrix equal to the correlation matrix of a stationary vector autoregression. However, just as in the univariate case, the Gaussian copula is unable to capture the volatility persistence exhibited by heteroskedastic time series. As an alternative, Beare and Seo (2015), Brechmann and Czado (2015) and Smith (2015) all suggest using vine copulas for (strongly) stationary series. We follow these authors and employ a D-vine, with pair-copula components of the form at Equation (4) to capture heteroskedasticity. We outline this below, although refer to Smith (2015) for further details on the specification of the vine and its time series properties.

When the multivariate series is (strongly) stationary and Markov of lag $p$, the D-vine copula density can be written as

$$
c(\boldsymbol{u})=K_{0}\left(\boldsymbol{u}_{1}\right) \prod_{t=2}^{T}\left(K_{0}\left(\boldsymbol{u}_{t}\right) \prod_{k=1}^{\min (t-1, p)} K_{k}\left(\boldsymbol{u}_{t-k}, \ldots, \boldsymbol{u}_{t}\right)\right) .
$$

The functionals $K_{0}, \ldots, K_{p}$ are each products of blocks of pair-copula densities, and do not vary with $t$ for stationary series. They are defined as

$$
K_{k}\left(\boldsymbol{u}_{t-k}, \ldots, \boldsymbol{u}_{t}\right)= \begin{cases}\prod_{l_{1}=1}^{m} \prod_{l_{2}=1}^{l_{1}-1} c_{l_{2}, l_{1}}^{(0)}\left(u_{j \mid i-1}, u_{i \mid j+1} ; \gamma_{l_{2}, l_{1}}^{(0)}\right) & \text { if } k=0 \\ \prod_{l_{1}=1}^{m} \prod_{l_{2}=1}^{m} c_{l_{2}, l_{1}}^{(k)}\left(u_{j \mid i-1}, u_{i \mid j+1} ; \gamma_{l_{2}, l_{1}}^{(k)}\right) & \text { if } 1 \leq k \leq p\end{cases}
$$

where $c_{l_{2}, l_{1}}^{(k)}$ is a bivariate pair-copula density with parameters $\gamma_{l_{2}, l_{1}}^{(k)}$. When $k=0$, there are $m(m-1) / 2$ of these associated with $K_{0}$, and they collectively capture cross-sectional dependence between the $m$ variables. For example, if they were each equal to the bivariate independence copula with density $c_{l_{2}, l_{1}}^{(0)}=1$, then $K_{0}=1$ and the variables would be independent at any given point in time. When $k>p$, there are $m^{2}$ pair-copulas associated with block $K_{k}$ that capture serial dependence at lag $k$. In total, there are $p\left(m^{2}\right)+m(m-1) / 2$ unique pair-copulas, which is much less than the $T m(T m-$ $1) / 2$ in an unconstrained D-vine. The indices of the pair-copula arguments are $i=l_{1}+m(t-1)$ and $j=l_{2}+m(t-k-1)$, and the argument values themselves $u_{i \mid j}, u_{j \mid i}$ are computed using the algorithm in Appendix C.2. Last, we note that if $m=1$, then $K_{0}=1, i=t, j=t-k$ and $K_{k}=$ $c_{1,1}^{(k)}\left(u_{t-k \mid t-1}, u_{t \mid t-k+1}\right)$, so that with the notation $c_{k+1} \equiv c_{1,1}^{(k)}$, the copula densities at Equations (5) and (9) are the same. 


\subsection{Estimation, Serial Dependence and Volatility Dependence}

Estimation is similar to the univariate case. The marginal distribution of each variable is estimated nonparametrically using adaptive kernel density estimation, from which the copula data are constructed. Equation (9) gives the likelihood, conditional on the copula data. It can be difficult to maximize for higher values of $m$ and $p$, so that we follow Min and Czado (2010) and Smith et al. (2010) and use MCMC to evaluate the posterior distribution. In the sampling scheme, the parameter vector of each unique pair-copula is generated jointly, conditional on the parameters of the other paircopulas. To do so, a Metropolis-Hastings step with an adaptive multivariate random walk Gaussian proposal (Roberts and Rosenthal, 2009) is used. Key to implementation is the efficient computation of the likelihood, as outlined in Appendix C.2.

Serial dependence in the series is summarized using measures of dependence between pairs $\left(y_{i, t-k}, y_{j, t}\right)$. These can be arranged into a $(m \times m)$ matrix for any given value of $k \geq 0$. Because the bivariate marginal copula between each pair is unavailable in closed form, we compute the metrics via Monte Carlo simulation from the vine copula. This can be undertaken efficiently using Algorithm 2 of Smith (2015). For example, pairwise Spearman's rho can be computed as

$$
\rho_{i, j, k}^{y}=12 E\left(u_{i, t-k} u_{j, t}\right)-3 \approx\left(\frac{12}{L} \sum_{l=1}^{L} u_{i, 1}^{[l]} u_{j, k+1}^{[l]}\right)-3,
$$

where $\left\{u_{i, t}^{[l]} ; i=1, \ldots, m, t=1, \ldots, k+1\right\}$ is an iterate from the joint distribution of $\left\{\boldsymbol{u}_{1}, \ldots, \boldsymbol{u}_{k+1}\right\}$, for $l=1, \ldots, L$. Then the matrix $P_{k}^{y}=\left\{\rho_{i, j, k}^{y}\right\}_{1 \leq i \leq m ; 1 \leq j \leq m}$ is a measure of overall $k$ th order serial dependence in the multivariate time series.

Similar dependence measures can be computed for the pair of volatility proxies $\left(v_{i, t-k}, v_{j, t}\right)$, where $v_{i, t}=V\left(y_{i, t}-\mu_{i, t}\right), \mu_{i, t}=E\left(y_{i, t}\right)$, and $V$ is the function defined in Section 2.2. While Theorem 1 is directly applicable here, the bivariate copula of $\left(v_{i, t-k}, v_{j, t}\right)$ cannot be computed in closed form because the underlying marginal copula of $\left(y_{i, t-k}, y_{j, t}\right)$ cannot either. Therefore, we again compute the dependence measures via Monte Carlo simulation, where iterates of the volatility proxies can be computed directly from those generated for the series. If Spearman's rho of the pair $\left(v_{i, t-k}, v_{j, t}\right)$ is denoted as $\rho_{i, j, k}^{v}$, then these values can be arranged into matrices $P_{k}^{v}=\left\{\rho_{i, j, k}^{v}\right\}_{1 \leq i \leq m ; 1 \leq j \leq m}$, for a given lag $k \geq 0$. The matrix $P_{0}^{v}$ measures cross-sectional dependence in volatility at a point in time, with the off-diagonal elements measuring volatility co-movement. For $k \geq 1$ the leading diagonal elements of $P_{k}^{v}$ are measures of own-series volatility persistence, whereas the off-diagonals are measures of volatility spillover. Volatility co-movement and spillover are widely documented in daily asset and exchange rate returns (Hamao et al., 1990, Baillie and Bollerslev, 1991), although multivariate GARCH models usually measure these through conditional moments, not marginally as we propose here.

\subsection{Multivariate Model of Exchange Rates}

We extend the analysis of the USD/AUD exchange rate in Section 2.3 to include the USD/EUR and USD/JPY rates, which are the two most traded currency pairs. As before, daily returns were computed using rates sourced from the FRED database which are synchronized to New York closing time. The USD/EUR and USD/JPY returns are both asymmetric (with skew 0.044 and 0.278 ) and fat-tailed (with kurtosis 5.23 and 7.22). Each margin is modeled nonparametrically using an adaptive kernel density estimator; see Figure 7. The D-vine copula at Equation (9) with $p=1$ is used to capture both cross-sectional and serial dependence simultaneously, with pair-copula densities given 
by $c^{M I X}$ at Equation (4). For the components of the mixture copula we use either all t copulas, or all convex Gumbels, resulting in two D-vines which we label 'Copula A' and 'Copula B', respectively. Both have a total of $12 \times 5=60$ parameters, and Table 7 reports their posterior means.

To summarize serial dependence in both the series and its volatility, the top of Table 8 reports the posterior mean estimates of the matrices $P_{k}^{y}$ and $P_{k}^{v}$ of pairwise marginal Spearman's rho. Results are reported for contemporaneous $(k=0)$ and first order serial $(k=1)$ dependence. Results are very similar for both Copulas $\mathrm{A}$ and $\mathrm{B}$, and we make a number of observations. First, crosssectional dependence in the returns $\left(P_{0}^{y}\right)$ is positive throughout, with that between the USD/AUD and USD/EUR currency pairs being the highest. Second, there is negligible first order serial dependence in the returns $\left(P_{1}^{y}\right)$. Third, there is co-movement in the volatility of the three series, with positive values on the off-diagonal of $P_{0}^{v}$. Fourth, there is volatility persistence in each series, with positive values along the leading diagonal of $P_{1}^{v}$. Last, there are positive volatility spillovers between series, as measured by the off-diagonal elements of $P_{1}^{v}$. All five features are consistent with previous studies of daily exchange rate returns (Baillie and Bollerslev, 2002, Nakatani and Teräsvirta, 2009).

We compare the dependence matrices with those computed from two trivariate GARCH models. These are the DCC-GARCH(1,1) (Engle, 2002) and BEKK(1,1) (Engle and Kroner, 1995) models. The diagonal form of the BEKK model is used because the likelihood for the full form is not logconcave for this series, which is a well-known problem. Table 8 also reports the dependence matrices for these two models, which are computed via simulation, and we make four observations. First, all models have positive return co-movements $P_{0}^{y}$, but are lowest for the DCC-GARCH(1,1). Second, volatility co-movements $P_{0}^{v}$ are also positive for all models, but are stronger for the copula models. Third, volatility persistence, given by the leading diagonal of $P_{1}^{v}$, is similar in size for all four models. Last, the major difference is that the off-diagonals of $P_{1}^{v}$ are positive for the copula models, but almost zero for the two GARCH models. Thus, first order volatility spillovers are indicated by the copula model, but are not by the multivariate GARCH models.

We extend the validation study to include these multivariate models, plus the BEKK $(1,0)$ model. We construct the one-day-ahead predictive distributions for the three returns series and the return on an equally-weighted currency portfolio. As in Section 2.3.2 we compute the mean number of exceedances $\hat{\alpha}$, and plots of these against the quantile $\alpha$ (given in the Online Appendix) suggest the copula models have more accurate VaR coverage than the multivariate GARCH models. For the equally-weighted currency portfolio, Table 9 reports exceedances for all five models and the results of the Christoffersen tests at six quantiles. As in the univariate case, the copula models dominate the multivariate GARCH models.

To illustrate the difference in density forecasts, we plot these for the USD/JPY return on three days in Figure 8. These are the days with the lowest and highest returns during the last 18 months in the data, along with the day with return closest to zero. Panels $(\mathrm{a}-\mathrm{c})$ plot the densities, while panels $(\mathrm{d}-\mathrm{f})$ plot their logarithm to better visualize the tails. For simplicity, we only plot the densities for Copula A, although for Copula B are similar. Note that the multivariate GARCH models are conditionally Gaussian, so that their predictive distributions are also. In contrast, the copula forecast densities in panels $(\mathrm{a}-\mathrm{c})$ are asymmetric, with skew coefficients of $0.113,0.473$ and 0.316 , respectively. Panels $(\mathrm{d}-\mathrm{f})$ show that the density forecasts from the copula model also have heavy tails, with a kurtosis of 5.78, 7.39 and 6.44, respectively. Clearly, the nonparametric margins combined with the copula function, translate into non-Gaussian predictive distributions. 


\section{Discussion}

Time series copula models are very general, in that all time series models have a copula specification. For example, Smith and Maneesoonthorn (2016) show how to compute the copula of a nonlinear state space model numerically. However, for many existing time series models — including popular models for heteroskedastic data - the time series copula cannot be written in closed form. Our approach is therefore an alternative copula specification to capture serial dependence for heteroskedastic data. For stationary first order Markov series the bivariate copula density has mass concentrated along the two diagonals of the unit cube, which mirrors that found empirically for two popular existing heteroskedastic models. It is extended to higher Markov orders and multivariate time series using Dvines. An important observation is that these vines are highly parsimonious, with densities that can be evaluated using $O(T)$ parallel algorithms. This enables the copula models to be readily estimated for the longer series encountered in practice.

The main theoretical result is the derivation of the bivariate copula of a volatility proxy at two time points. We find that the copula does not depend on the specific transformation $V$ used in Section 2.2 . For example, it is the same if the volatility proxy is either $v_{t}=\left|y_{t}-E\left(y_{t}\right)\right|$ or $v_{t}=\left(y_{t}-E\left(y_{t}\right)\right)^{2}$. The copula fully characterizes the (unconditional) dependence between $v_{t}$ and $v_{t-k}$ at a given lag $k \geq 1$. While it is a function of the time series copula, it is also a function of the marginal distribution of the data whenever that margin is asymmetric. This has an important implication for applied modeling: while the choice of $C$ in Equation (11) completely determines the serial dependence of the series, it does not always do so for volatility. We show how dependence metrics can be computed from the volatility copulas, which provide measures of volatility persistence, along with co-movement and spillover for multivariate series. These can be computed by simulation for any stationary time series, not just the copula model proposed here. They can be used to compare the degree of volatility dependence arising from different nonlinear time series models, as in Tables 4 and 8 .

A major advantage of copula models is the simplicity with which they incorporate complex margins; for example, the exchange rate returns series exhibit asymmetry and heavy tails. As noted by Chen and Fan (2006) and others, these can be accurately captured using nonparametric methods, and we show in Figure 8 that these affect the forecast densities substantitally. In comparison, most existing time series models are conditionally Gaussian or t distributed, and density forecasts are also; e.g. see Clark and Ravazzolo (2015). Moreover, the marginal distributions are often poorly calibrated, as illustrated in Figure 4. Ultimately, the VaR forecasts from the copula model are more accurate. We illustrate this using daily exchange rate returns with GARCH benchmark models in the univariate case, and BEKK and DCC benchmark models in the multivariate case. In the latter, our copula model also dominates the multivariate GARCH models for a portfolio of the three rates, indicating that the copula also captures the cross-sectional dependence accurately.

\section{Appendix A D-vine Copula Density}

In this appendix we outline the derivation of the D-vine copula density at Equation (5). The copula density of a Markov $p$ process can written as

$$
c^{D V}(\boldsymbol{u})=\prod_{t=2}^{T} f\left(u_{t} \mid u_{\max (1, t-p)}, \ldots, u_{t-1}\right),
$$


where $f\left(u_{1}\right)=1$ because the marginal distribution of $u_{1}$ is uniform on $[0,1]$. For $t-p \leq s<t$, there always exists a density $c_{t, s}$ on $[0,1]^{2}$ such that

$$
\begin{aligned}
& f\left(u_{t}, u_{s} \mid u_{t-1}, \ldots, u_{s+1}\right)=f\left(u_{t} \mid u_{t-1}, \ldots, u_{s+1}\right) f\left(u_{s} \mid u_{t-1}, \ldots, u_{s+1}\right) \\
& \quad \times \quad c_{t, s}\left(F\left(u_{s} \mid u_{t-1}, \ldots, u_{s+1}\right), F\left(u_{t} \mid u_{t-1}, \ldots, u_{s+1}\right) ; u_{t-1}, \ldots, u_{s+1}\right),
\end{aligned}
$$

which is the theorem of Sklar applied conditional on $u_{t-1}, \ldots, u_{s+1}$. In a vine copula, $c_{t, s}$ is a bivariate pair-copula density, and it is simplified by dropping dependence on $\left(u_{t-1}, \ldots, u_{s+1}\right)$. The pair-copula captures the dependence between $y_{t}$ and $y_{s}$, conditional on the intervening observations. Denoting $u_{s \mid t-1}=F\left(u_{s} \mid u_{t-1}, \ldots, u_{s+1}\right)$ and $u_{t \mid s+1}=F\left(u_{t} \mid u_{t-1}, \ldots, u_{s+1}\right)$, the above gives $f\left(u_{t} \mid u_{t-1}, \ldots, u_{s}\right)=$ $c_{t, s}\left(u_{s \mid t-1}, u_{t \mid s+1}\right) f\left(u_{t} \mid u_{t-1}, \ldots, u_{s+1}\right)$. Repeated application of the above with $s=\max (1, t-p), \ldots, t-$ 1 gives

$$
\begin{aligned}
f\left(u_{t} \mid u_{\max (1, t-p)}, \ldots, u_{t-1}\right) & =\prod_{s=\max (1, t-p)}^{t-1} c_{t, s}\left(u_{s \mid t-1}, u_{t \mid s+1}\right) \\
& =\prod_{k=1}^{\min (p, t-1)} c_{t, t-k}\left(u_{t-k \mid t-1}, u_{t \mid t-k+1}\right)
\end{aligned}
$$

where we set $s=t-k$. If the series is stationary, it is straightforward - for example, see Smith (2015) - to show that the pair-copulas $c_{t, t-k}$ are invariant with respect to $t$, so that we can write $c_{t, t-k}=c_{k+1}$ throughout, resulting in Equation (5).

Last, we note that compared to Equation (2.4) of Smith et al. (2010), the order of the two arguments of each pair-copula is switched. While this is unimportant when the pair-copula is symmetric, it is when the pair-copula is asymmetric, as with the mixture copula $c^{M I X}$ here. It is particularly important to keep note of the order of the arguments of the pair-copulas when implementing the algorithms in Appendix C.

\section{Appendix B Copula of Transformed Variables}

Consider two continuous random variables $Y_{1}$ and $Y_{2}$, with joint distribution function $F$, bivariate copula function $\bar{C}$ and density $\bar{c}$, and marginal distribution functions $F_{1}$ and $F_{2}$, respectively. (In Theorem 1 and Lemma 1, these random variables correspond to the time series at times $s$ and $t$, respectively.) In this appendix we derive the bivariate copula function $C_{V}$ of $V_{1}=V\left(Y_{1}-\mu_{1}\right)$ and $V_{2}=V\left(\overline{Y_{2}}-\mu_{2}\right)$, where $V: \mathbb{R} \rightarrow \mathbb{R}^{+}$is the transformation defined in Section 2.2, $\mu_{1}=E\left(Y_{1}\right)$ and $\mu_{2}=E\left(Y_{2}\right)$. We show that, in general, $C_{V}$ is a function of both $\bar{C}$, and also the marginals $F_{1}$ and $F_{2}$. We also derive the copula density $c_{V}$ of $V_{1}$ and $V_{2}$. We consider separately the special case where both $Y_{1}$ and $Y_{2}$ are strictly symmetrically distributed.

\section{B.1 General Marginals Case}

Let $G(V(a))=|a|$ for any $a \in \mathbb{R}$, and $G($.$) is a differentiable function function, where G: \mathbb{R}^{+} \rightarrow \mathbb{R}^{+}$. Recognizing that $G\left(v_{j}\right)=G\left(V\left(y_{j}-\mu_{j}\right)\right)=\left|y_{j}-\mu_{j}\right|$, the values of $v_{j}$ can be mapped to $y_{j}$ (in a one to two mapping), by the identity $y_{j}=(-1)^{i} G\left(v_{j}\right)+\mu_{j}$, for $i \in\{1,2\}$. Since this mapping is deterministic, through the $G$ function, the joint distribution of $\left(V_{1}, V_{2}\right)$ can be derived from the joint distribution of $\left(Y_{1}, Y_{2}\right)$ :

$$
F_{V}\left(v_{1}, v_{2}\right)=\operatorname{Pr}\left(-G\left(v_{1}\right)<Y_{1}-\mu_{1}<G\left(v_{1}\right),-G\left(v_{2}\right)<Y_{2}-\mu_{2}<G\left(v_{2}\right)\right)
$$




$$
=\sum_{i=1}^{2} \sum_{j=1}^{2}(-1)^{i}(-1)^{j} F\left((-1)^{i} G\left(v_{1}\right)+\mu_{1},(-1)^{j} G\left(v_{2}\right)+\mu_{2}\right) \text {. }
$$

Further, by Sklar's Theorem, $F\left(y_{1}, y_{2}\right)=\bar{C}\left(F_{1}\left(y_{1}\right), F_{2}\left(y_{2}\right)\right)$, so $F_{V}\left(v_{1}, v_{2}\right)$ can be written as a function of $\bar{C}\left(F_{1}\left(y_{1}\right), F_{2}\left(y_{2}\right)\right)$ as

$$
F_{V}\left(v_{1}, v_{2}\right)=\sum_{i=1}^{2} \sum_{j=1}^{2}(-1)^{i}(-1)^{j} \bar{C}\left(F_{1}\left((-1)^{i} G\left(v_{1}\right)+\mu_{1}\right), F_{2}\left((-1)^{j} G\left(v_{2}\right)+\mu_{2}\right)\right) .
$$

With the marginal distribution function of $V_{j}$ denoted by $F_{V_{j}}$ and the corresponding copula datum $\tilde{u}_{j}=F_{V_{j}}\left(v_{j}\right)$, inverting Sklar's theorem yields the copula function

$$
\begin{aligned}
& C_{V}\left(\tilde{u}_{1}, \tilde{u}_{2}\right)=F_{V}\left(F_{V_{1}}^{-1}\left(\tilde{u}_{1}\right), F_{V_{2}}^{-1}\left(\tilde{u}_{2}\right)\right) \\
& =\sum_{i=1}^{2} \sum_{j=1}^{2}(-1)^{i}(-1)^{j} \bar{C} \\
& \left.\quad F_{1}\left((-1)^{i} G\left(F_{V_{1}}^{-1}\left(\tilde{u}_{1}\right)\right)+\mu_{1}\right), F_{2}\left((-1)^{j} G\left(F_{V_{2}}^{-1}\left(\tilde{u}_{2}\right)\right)+\mu_{2}\right)\right), \\
& F_{V_{j}}\left(v_{j}\right)=\operatorname{Pr}\left(V_{j}<v_{j}\right)=\operatorname{Pr}\left(-G\left(v_{j}\right)<Y_{j}-\mu_{j}<G\left(v_{j}\right)\right) \\
& =F_{j}\left(G\left(v_{j}\right)+\mu_{j}\right)-F_{j}\left(-G_{j}\left(v_{j}\right)+\mu_{j}\right) .
\end{aligned}
$$

The quantile function $F_{V_{j}}^{-1}$ can be obtained by numerically inverting (11) for any given marginal $F_{j}$.

The copula density can be obtained by differentiating the copula function in (10):

$$
\begin{gathered}
c_{V}\left(\tilde{u}_{1}, \tilde{u}_{2}\right)=\frac{\partial^{2}}{\partial \tilde{u}_{1} \partial \tilde{u}_{2}} C_{V}\left(\tilde{u}_{1}, \tilde{u}_{2}\right) \\
=\sum_{i=1}^{2} \sum_{j=1}^{2} \bar{c}\left(F_{1}\left(\mu_{1}+(-1)^{i} G\left(F_{V_{1}}^{-1}\left(\tilde{u}_{1}\right)\right)\right), F_{2}\left(\mu_{2}+(-1)^{j} G\left(F_{V_{2}}^{-1}\left(\tilde{u}_{2}\right)\right)\right)\right) f_{1}\left(\mu_{1}+(-1)^{i} G\left(F_{V_{1}}^{-1}\left(\tilde{u}_{1}\right)\right)\right) \times \\
\frac{f_{2}\left(\mu_{2}+(-1)^{j} G\left(F_{V_{2}}^{-1}\left(\tilde{u}_{2}\right)\right)\right) G^{\prime}\left(F_{V_{1}}^{-1}\left(\tilde{u}_{1}\right)\right) G^{\prime}\left(F_{V_{2}}^{-1}\left(\tilde{u}_{2}\right)\right)}{f_{V_{1}}\left(F_{V_{1}}^{-1}\left(\tilde{u}_{1}\right)\right) f_{V_{2}}\left(F_{V_{2}}^{-1}\left(\tilde{u}_{2}\right)\right)} \\
=\frac{\sum_{i=1}^{2} \frac{\sum_{j=1}^{2} f\left(\mu_{1}+(-1)^{i} G\left(F_{V_{1}}^{-1}\left(\tilde{u}_{1}\right)\right), \mu_{2}+(-1)^{j} G\left(F_{V_{2}}^{-1}\left(\tilde{u}_{2}\right)\right)\right) G^{\prime}\left(F_{V_{1}}^{-1}\left(\tilde{u}_{1}\right)\right) G^{\prime}\left(F_{V_{2}}^{-1}\left(\tilde{u}_{2}\right)\right)}{f_{V_{1}}\left(F_{V_{1}}^{-1}\left(\tilde{u}_{1}\right)\right) f_{V_{2}}\left(F_{V_{2}}^{-1}\left(\tilde{u}_{2}\right)\right)}}{}
\end{gathered}
$$

with

$$
f_{V_{j}}\left(v_{j}\right)=\frac{d}{d v_{j}} F_{V_{j}}\left(v_{j}\right)=\left(f_{j}\left(G\left(v_{j}\right)+\mu_{j}\right)+f_{j}\left(-G\left(v_{j}\right)+\mu_{j}\right)\right) G^{\prime}\left(v_{j}\right) .
$$

\section{B.2 Symmetric Marginals Case}

In the special case where the marginal distributions $F_{1}$ and $F_{2}$ are both symmetric around their respective means, we have that $F_{j}\left(-G\left(v_{j}\right)+\mu_{j}\right)=1-F_{j}\left(G\left(v_{j}\right)+\mu_{j}\right)$, for $j=\{1,2\}$. Applying this relation to Equation (11), gives

$$
F_{V_{j}}\left(v_{j}\right)=2 F_{j}\left(G\left(v_{j}\right)+\mu_{j}\right)-1 .
$$

By substituting $F_{V_{j}}\left(v_{j}\right)=\tilde{u}_{j}$ and $v_{j}=F_{V_{j}}^{-1}\left(\tilde{u}_{j}\right)$, along with simple rearrangements,

$$
G\left(F_{V_{j}}^{-1}\left(\tilde{u}_{j}\right)\right)=F_{j}^{-1}\left(\frac{1+\tilde{u}_{j}}{2}\right)-\mu_{j}
$$


Since the marginal distribution is symmetric around $\mu_{j}$, we also have that

$$
-G\left(F_{V_{j}}^{-1}\left(\tilde{u}_{j}\right)\right)=F_{j}^{-1}\left(\frac{1-\tilde{u}_{j}}{2}\right)-\mu_{j} .
$$

Substituting the simplified expressions for $G\left(F_{V_{j}}^{-1}\left(\tilde{u}_{j}\right)\right)$ and $-G\left(F_{V_{j}}^{-1}\left(\tilde{u}_{j}\right)\right)$ into Equation (10) gives

$$
C_{V}\left(\tilde{u}_{1}, \tilde{u}_{2}\right)=\sum_{i=1}^{2} \sum_{j=1}^{2}(-1)^{i}(-1)^{j} \bar{C}\left(\frac{1+(-1)^{i} \tilde{u}_{1}}{2}, \frac{1+(-1)^{j} \tilde{u}_{2}}{2}\right) \text {. }
$$

Finally, by differentiating the copula distribution above, the copula density is

$$
c_{V}\left(\tilde{u}_{1}, \tilde{u}_{2}\right)=\sum_{i=1}^{2} \sum_{j=1}^{2} \frac{1}{4} \bar{c}\left(\frac{1+(-1)^{i} \tilde{u}_{1}}{2}, \frac{1+(-1)^{j} \tilde{u}_{2}}{2}\right) .
$$

Note that the copula function of the transformed variable in this special case, where both margins are symmetric around $\mu_{j}$, does not depend on the form of the marginal distribution $F_{j}$.

\section{Appendix C Efficient Likelihood Evaluation}

Computing the two D-vine copula densities at Equations (5) and (9) requires efficient evaluation of the arguments of the pair-copulas. In this appendix we outline algorithms to compute these. The algorithms are extensions of that orginally proposed by Aas et al. (2009), and further developed in Smith et al. (2010) and Smith (2015). They differ in three ways: (i) they are re-ordered so that the computations can be undertaken in parallel; (ii) they exploit the parsimonious structures of the two vine copulas; and (iii) they are based on recursions that account for the pair-copulas being mixtures of possibly asymmetric copulas.

\section{C.1 Univariate Series}

The arguments of the pair-copulas can be computed by exploiting the recursive relationships

$$
u_{t \mid s}=h_{s, t}^{1}\left(u_{t \mid s+1} \mid u_{s \mid t-1}\right), \text { and } u_{s \mid t}=h_{s, t}^{2}\left(u_{s \mid t-1} \mid u_{t \mid s+1}\right) \text {, }
$$

where, for the specific vine in Equation (5), if $k=t-s$ then

$$
h_{s, t}^{1}(v \mid u)=\frac{\partial}{\partial u} C_{k+1}\left(u, v ; \gamma_{k+1}\right), \text { and } h_{s, t}^{2}(u \mid v)=\frac{\partial}{\partial v} C_{k+1}\left(u, v ; \gamma_{k+1}\right) .
$$

Here, $C_{k+1}\left(u, v ; \gamma_{k+1}\right)=\int_{0}^{u} \int_{0}^{v} c_{k+1}(\tilde{u}, \tilde{v} ; \gamma) d \tilde{u} d \tilde{v}$ is the pair-copula function for $k=1,2, \ldots, p$. We note that these recursions are more general than those given in Smith et al. (2010). These authors assume that $h_{s, t}^{1}=h_{s, t}^{2}$, which is true for the pair-copula types they examine. However, this is not the case when the pair-copula is the mixture copula with function $C^{M I X}$, with the partial derivatives given in Table 1.

The $O\left(T^{2}\right)$ algorithms in Aas et al. (2009) and Smith et al. (2010) compute and store all $T(T-1)$ values $\left\{u_{t \mid s}, u_{s \mid t} ; 1 \leq t \leq T, s<t\right\}$, which is impractical for high values of $T$. However, to compute the likelihood in Equation (5) , only the values $\mathcal{U}=\left\{u_{t \mid s}, u_{s \mid t} ; 1 \leq t \leq T, \max (1, t-p) \leq s<t\right\}$ need computing and storing. Moreover, we evaluate the elements of $\mathcal{U}$ in a different order to allow the computations to be undertaken in parallel, as follows: 


\section{Algorithm 1.}

For $t=1, \ldots, T$ :

Step (1). Set $u_{t \mid t}=u_{t}$.

For $k=1, \ldots, p$ :

For $t=k+1, \ldots, T$ (compute inner loop in parallel):

Step (2.1). $u_{t \mid t-k}=h_{t-k, t}^{1}\left(u_{t \mid t-k+1} \mid u_{t-k \mid t-1}\right)$

Step (2.2). $u_{t-k \mid t}=h_{t-k, t}^{2}\left(u_{t-k \mid t-1} \mid u_{t \mid t-k+1}\right)$

Once computed, the elements in $\mathcal{U}$ need to be stored efficiently. It is possible to store these in a $(T \times T)$ matrix, with $u_{t \mid s}$ stored in element $(t, s)$, and $u_{s \mid t}$ in element $(s, t)$. However, this is prohibitive for longer time series. Instead, $\mathcal{U}$ can be stored efficiently either as a banded matrix with bandwidth $p$, or a $(T \times p \times 2)$ array, with $u_{t \mid t-k}$ stored as element $(t, k, 1)$, and $u_{t-k \mid t}$ as element $(t, k, 2)$. We use the latter approach in our code.

\section{C.2 Multivariate Series}

For the vine copula at Equation (9), there is a one-to-one relationship between the indices $\left(s, t, l_{1}, l_{2}\right)$, and those of the pair-copula arguments $(i, j)$. To evaluate these arguments we use the recursive relationships

$$
u_{i \mid j}=h_{j, i}^{1}\left(u_{i \mid j+1} \mid u_{j \mid i-1}\right) \text {, and } u_{j \mid i}=h_{j, i}^{2}\left(u_{j \mid i-1} \mid u_{i \mid j+1}\right) .
$$

The functions are

$$
h_{j, i}^{1}(v \mid u)=\frac{\partial}{\partial u} C_{l_{2}, l_{1}}^{(k)}\left(u, v ; \gamma_{l_{2}, l_{1}}^{(k)}\right), \text { and } h_{j, i}^{2}(u \mid v)=\frac{\partial}{\partial v} C_{l_{2}, l_{1}}^{(k)}\left(u, v ; \gamma_{l_{2}, l_{1}}^{(k)}\right),
$$

where $s=\lceil j / m\rceil, t=\lceil i / m\rceil, k=t-s, l_{1}=i-m(t-1), l_{2}=j-m(s-1)$, and the pair-copula function $C_{l_{2}, l_{1}}^{(k)}\left(u, v ; \gamma_{l_{2}, l_{1}}^{(k)}\right)=\int_{0}^{u} \int_{0}^{v} c_{l_{2}, l_{1}}^{(k)}\left(\tilde{u}, \tilde{v} ; \gamma_{l_{2}, l_{1}}^{(k)}\right) d \tilde{u} d \tilde{v}$. As in the univariate case, we employ $C^{M I X}$ for the pair-copula functions, so that the partial derivatives required to compute $h_{i, j}^{1}$ and $h_{j, i}^{2}$ above are given in Table 1. Following (Smith, 2015), we note that only the values

$$
\mathcal{U}=\left\{u_{i \mid j}, u_{j \mid i} ; 1 \leq i \leq T m, \max (1, m(\lceil i / m\rceil-1-p)+1) \leq j<i\right\}
$$

are needed to compute the likelihood. These can be computed using the $O\left(p m^{2} T\right)$ algorithm below. Algorithm 2.

For $t=1, \ldots, T, l=1, \ldots, m$ :

Step (1,1). Set $i=t+(l-1) m$.

Step (1.2). Set $u_{i \mid i}=u_{l, t}$.

For $r=1, \ldots,(p+1) m-1$ :

For $i=r+1, \ldots, m T$ (compute inner loop in parallel):

Step (2.1). Set $j=i-r, s=\lceil j / m\rceil, t=\lceil i / m\rceil, k=t-s, l_{1}=i-m(t-1), l_{2}=j-m(s-1)$.

Step (2.2). Compute $u_{i \mid j}=h_{j, i}^{1}\left(u_{i \mid j+1} \mid u_{j \mid i-1}\right)$.

Step (2.3). Compute $u_{j \mid i}=h_{j, i}^{2}\left(u_{j \mid i-1} \mid u_{i \mid j+1}\right)$.

The arguments in $\mathcal{U}$ are efficiently stored in a 3-dimensional $(\operatorname{Tm} \times(m(p+1)-1) \times 2)$ array, with $u_{j \mid i}$ stored as element $(i, i-j, 1)$, and $u_{j \mid i}$ as element $(i, i-j, 2)$. 


\section{References}

Aas, K., Czado, C., Frigessi, A., and Bakken, H. (2009). Pair-copula constructions of multiple dependence. Insurance: Mathematics and Economics, 44(2):182 - 198.

Almeida, C. and Czado, C. (2012). Efficient Bayesian inference for stochastic time-varying copula models. Computational Statistics 85 Data Analysis, 56(6):1511-1527.

Baillie, R. T. and Bollerslev, T. (1991). Intra-day and inter-market volatility in foreign exchange rates. The Review of Economic Studies, 58(3):565-585.

Baillie, R. T. and Bollerslev, T. (2002). The message in daily exchange rates: a conditional-variance tale. Journal of Business \& Economic Statistics, 20(1):60-68.

Beare, B. K. (2010). Copulas and Temporal Dependence. Econometrica, 78(1):395-410.

Beare, B. K. (2012). Archimedean copulas and temporal dependence. Econometric Theory, 28(06):1165-1185.

Beare, B. K. and Seo, J. (2015). Vine copula specifications for stationary multivariate Markov chains. Journal of Time Series Analysis, 36(2):228-246.

Biller, B. (2009). Copula-based multivariate input models for stochastic simulation. Operations Research, 57(4):878-892.

Biller, B. and Nelson, B. (2003). Modeling and generating multivariate time-series input processes using a vector autoregressive technique. ACM Transactions on Modeling and Computer Simulation, 13(3):1049-3301.

Boothe, P. and Glassman, D. (1987). The statistical distribution of exchange rates: empirical evidence and economic implications. Journal of International Economics, 22(3):297-319.

Brechmann, E. C. and Czado, C. (2015). COPAR- multivariate time series modeling using the copula autoregressive model. Applied Stochastic Models in Business and Industry, 31(4):495-514.

Brockwell, P. J. and Davis, R. A. (1991). Time Series : Theory and Methods. Springer Series in Statistics. Springer, New York (N.Y.).

Celeux, G., Forbes, F., Robert, C. P., and Titterington, D. M. (2006). Deviance information criteria for missing data models. Bayesian analysis, 1(4):651-673.

Chen, X. and Fan, Y. (2006). Estimation of copula-based semiparametric time series models. Journal of Econometrics, 130(2):307-335.

Chen, X., Wu, W. B., and Yi, Y. (2009). Efficient Estimation of Copula-based Semiparametric Markov Models. Annals of statistics, 37(6B):4214-4253.

Christoffersen, P. F. (1998). Evaluating interval forecasts. International Economic Review, 39:841862.

Clark, T. E. and Ravazzolo, F. (2015). Macroeconomic forecasting performance under alternative specifications of time-varying volatility. Journal of Applied Econometrics, 30(4):551-575. 
Creal, D. D. and Tsay, R. S. (2015). High dimensional dynamic stochastic copula models. Journal of Econometrics, 189(2):335-345.

Darsow, W. F., Nguyen, B., and Olsen, E. T. (1992). Copulas and Markov processes. Illinois Journal of Mathematics, 36(4):600-642.

De Lira Salvatierra, I. and Patton, A. J. (2015). Dynamic copula models and high frequency data. Journal of Empirical Finance, 30:120-135.

Demarta, S. and McNeil, A. J. (2005). The t copula and related copulas. International Statistical Review/Revue Internationale de Statistique, pages 111-129.

Domma, F., Giordano, S., and Perri, P. F. (2009). Statistical modeling of temporal dependence in financial data via a copula function. Communications in Statistics - Simulation and Computation, 38(4):703-728.

Engle, R. (2002). Dynamic conditional correlation: A simple class of multivariate generalized autoregressive conditional heteroskedasticity models. Journal of Business $\&$ Economic Statistics, 20(3):339-350.

Engle, R. F. and Kroner, K. F. (1995). Multivariate simultaneous generalized ARCH. Econometric Theory, 11(01):122-150.

Fortin, I. and Kuzmics, C. (2002). Tail-dependence in stock-return pairs. Intelligent Systems in Accounting, Finance and Management, 11(2):89-107.

Hafner, C. M. and Manner, H. (2012). Dynamic stochastic copula models: Estimation, inference and applications. Journal of Applied Econometrics, 27(2):269-295.

Hamao, Y., Masulis, R. W., and Ng, V. (1990). Correlations in price changes and volatility across international stock markets. Review of Financial studies, 3(2):281-307.

Hansen, P. R. and Lunde, A. (2005). A forecast comparison of volatility models: does anything beat a GARCH(1,1)? Journal of Applied Econometrics, 20(7):873-889.

Ibragimov, R. (2009). Copula-based characterizations for higher order markov processes. Econometric Theory, 25(03):819-846.

Joe, H. (1997). Multivariate models and multivariate dependence concepts. CRC Press.

Joe, H. (2014). Dependence Modeling with Copulas. Chapman and Hall/CRC.

Junker, M. and May, A. (2005). Measurement of aggregate risk with copulas. The Econometrics Journal, 8(3):428-454.

Lambert, P. and Vandenhende, F. (2002). A copula-based model for multivariate non-normal longitudinal data: analysis of a dose titration safety study on a new antidepressant. Statistics in Medicine, 21(21):3197-3217.

Min, A. and Czado, C. (2010). Bayesian inference for multivariate copulas using pair-copula constructions. Journal of Financial Econometrics, 8(4):511-546. 
Nakatani, T. and Teräsvirta, T. (2009). Testing for volatility interactions in the constant conditional correlation GARCH model. The Econometrics Journal, 12(1):147-163.

Nelsen, R. B. (2006). An Introduction to Copulas (Springer Series in Statistics). Springer-Verlag New York, Inc., Secaucus, NJ, USA.

Oh, D. H. and Patton, A. J. (2013). Time-varying systemic risk: evidence from a dynamic copula model of CDS spreads. Economic Research Initiatives at Duke (ERID) Working Paper, (167).

Oh, D. H. and Patton, A. J. (2016). High-dimensional copula-based distributions with mixed frequency data. Journal of Econometrics.

Patton, A. J. (2006). Modelling asymmetric exchange rate dependence. International Economic Review, 47(2):527-556.

Patton, A. J. (2012). A review of copula models for economic time series. Journal of Multivariate Analysis, 110:4-18.

Rémillard, B., Papageorgiou, N., and Soustra, F. (2012). Copula-based semiparametric models for multivariate time series. Journal of Multivariate Analysis, 110:30-42.

Roberts, G. O. and Rosenthal, J. S. (2009). Examples of adaptive MCMC. Journal of Computational and Graphical Statistics, 18(2):349-367.

Shimazaki, H. and Shinomoto, S. (2010). Kernel bandwidth optimization in spike rate estimation. J. Comput. Neurosci., 29(1-2):171-182.

Sklar, A. (1959). Fonctions de Répartition À N Dimensions Et Leurs Marges. Université Paris 8.

Smith, M., Min, A., Almeida, C., and Czado, C. (2010). Modeling longitudinal data using a pair-copula decomposition of serial dependence. Journal of the American Statistical Association, 105(492):1467-1479.

Smith, M. S. (2015). Copula modelling of dependence in multivariate time series. International Journal of Forecasting, 31(3):815 - 833 .

Smith, M. S. and Maneesoonthorn, W. (2016). Inversion copulas from nonlinear state space models. Working Paper.

Smith, M. S. and Vahey, S. P. (2016). Asymmetric density forecasts for US macroeconomic variables from a Gaussian copula model of cross-sectional and serial dependence. Journal of Business and Economic Statistics, 34(3):416-434. 


\begin{tabular}{l}
\hline \hline Bivariate Mixture Copula (Parameters $\left.\gamma=\left\{w, \gamma^{a}, \gamma^{b}\right\}\right)$ \\
(i) Copula Function \\
$C^{M I X}(u, v ; \gamma)=w C^{a}\left(u, v ; \gamma^{a}\right)+(1-w)\left(v-C^{b}\left(1-u, v ; \gamma^{b}\right)\right)$ \\
(ii) Partial Derivatives \\
$h^{M I X, 1}(v \mid u ; \gamma) \equiv \frac{\partial}{\partial u} C^{M I X}(u, v ; \gamma)=w h^{a, 1}\left(v \mid u ; \gamma^{a}\right)+(1-w) h^{b, 1}\left(v \mid 1-u ; \gamma^{b}\right)$ \\
$h^{M I X, 2}(u \mid v ; \gamma) \equiv \frac{\partial}{\partial v} C^{M I X}(u, v ; \gamma)=w h^{a, 2}\left(u \mid v ; \gamma^{a}\right)+(1-w)\left(1-h^{b, 2}\left(1-u \mid v ; \gamma^{b}\right)\right)$ \\
where $h^{x, 2}\left(u \mid v ; \gamma^{x}\right) \equiv \frac{\partial}{\partial v} C^{x}\left(u, v ; \gamma^{x}\right)$ and $h^{x, 1}\left(v \mid u ; \gamma^{x}\right) \equiv \frac{\partial}{\partial u} C^{x}\left(u, v ; \gamma^{x}\right)$ for $x=a, b$ \\
\hline Convex Gumbel Copula $(\operatorname{Parameters} 0 \leq \delta \leq 1, \tau \geq 0)$ \\
(i) Copula Function \\
$C^{c G}(u, v ; \tau, \delta)=\delta C^{G}(u, v ; \tau)+(1-\delta)\left(u+v-1+C^{G}(1-u, 1-v ; \tau)\right)$ \\
(ii) Partial Derivatives \\
$h^{c G}(v \mid u ; \tau, \delta) \equiv \frac{\partial}{\partial u} C^{c G}(u, v ; \tau, \delta)=\delta h^{G}(v \mid u ; \tau)+(1-\delta)\left(1-h^{G}(1-v \mid 1-u ; \tau)\right)$ \\
$h^{c G}(u \mid v ; \tau, \delta) \equiv \frac{\partial}{\partial v} C^{c G}(u, v ; \tau, \delta)=\delta h^{G}(u \mid v ; \tau)+(1-\delta)\left(1-h^{G}(1-u \mid 1-v ; \tau)\right)$ \\
where $h^{G}(u \mid v ; \tau) \equiv \frac{\partial}{\partial v} C^{G}(u, v ; \tau)=\frac{\partial}{\partial v} C^{G}(v, u ; \tau)$ \\
\hline \hline
\end{tabular}

Table 1: Distribution functions and their partial derivatives for the mixture copula with density $c^{M I X}(u, v ; \gamma)$, and the convex Gumbel copula with density $c^{c G}(u, v ; \tau, \delta)$. Here, $C^{G}$ is the Gumbel copula function parameterized in terms of Kendall's tau $\tau$. We note that when $C^{a}$ or $C^{b}$ are t-copulas, their partial derivatives can be found in Aas et al. (2009).

\begin{tabular}{ccccccccc}
\hline & \multicolumn{2}{c}{$\rho_{1}^{y}$} & \multicolumn{2}{c}{$\rho_{1}^{v}$} & $r_{1}^{y}$ & $r_{1}^{|y|}$ & $r_{1}^{y^{2}}$ \\
\hline \multicolumn{2}{c}{ Case } & Copula & Empirical & Copula & Empirical & & & \\
\hline ARCH & $\left(\alpha_{1}=0.5\right)$ & -0.002 & -0.003 & 0.241 & 0.240 & -0.007 & 0.373 & 0.451 \\
& $(0.022)$ & $(0.005)$ & $(0.075)$ & $(0.005)$ & $(0.005)$ & $(0.005)$ & $(0.005)$ \\
& $\left(\alpha_{1}=0.9\right)$ & -0.001 & -0.002 & 0.393 & 0.371 & -0.014 & 0.655 & 0.574 \\
& $(0.013)$ & $(0.005)$ & $(0.115)$ & $(0.006)$ & $(0.005)$ & $(0.005)$ & $(0.005)$ \\
\hline \multicolumn{2}{c}{$\left(\phi_{1}=0.5\right)$} & -0.004 & -0.004 & 0.186 & 0.230 & -0.005 & 0.221 & 0.102 \\
& $(0.025)$ & $(0.005)$ & $(0.018)$ & $(0.005)$ & $(0.005)$ & $(0.005)$ & $(0.005)$ \\
& $\left(\phi_{1}=0.9\right)$ & -0.002 & -0.004 & 0.395 & 0.453 & -0.026 & 0.397 & 0.142 \\
& $(0.293)$ & $(0.005)$ & $(0.206)$ & $(0.005)$ & $(0.005)$ & $(0.005)$ & $(0.005)$ \\
\hline
\end{tabular}

Table 2: Spearman's rho of first order serial dependence in the level $\left(\rho_{1}^{y}\right)$ and volatility $\left(\rho_{1}^{v}\right)$ for four datasets simulted from $\mathrm{ARCH}(1)$ and $\mathrm{SV}(1)$ models. Columns labelled 'Copula' show values for the fitted time series copula model, where $c_{2}=c^{M I X}$ with $c^{a}$ and $c^{b}$ given by $\mathrm{t}$ copula densities. Columns labelled 'Empirical' show nonparametric empirical values. Standard errors are given below in parentheses. For comparison, the final three columns report the first order sample autocorrelations for the three series $\left\{y_{t}\right\},\left\{\left|y_{t}\right|\right\}$ and $\left\{y_{t}^{2}\right\}$. 


\begin{tabular}{lccccc}
\hline Parameter & $\zeta^{a}>0$ & $\nu^{a}$ & $\zeta^{b}>0$ & $\nu^{b}$ & $w$ \\
\hline Posterior Mean & 0.153 & 12.101 & 0.173 & 10.325 & 0.482 \\
Posterior Interval & $(0.008,0.508)$ & $(3.910,31.143)$ & $(0.016,0.511)$ & $(3.821,25.387)$ & $(0.038,0.945)$ \\
MLE & 0 & 39.995 & 0.020 & 4.777 & 0.321 \\
SE & $(0.339)$ & $(0.171)$ & $(0.172)$ & $(0.502)$ & $(0.164)$ \\
Conf. Interval & $(0,0.559)$ & $(39.721,40.269)$ & $(0,0.295)$ & $(3.974,5.580)$ & $(0.059,0.583)$ \\
\hline Metric & $\lambda_{\text {low }}^{y}=\lambda_{\text {up }}^{y}$ & $\rho_{1}^{y}$ & $\rho_{1}^{v}$ & $\lambda_{\text {low }}^{v}(0.05)$ & $\lambda_{\text {up }}^{v}(0.05)$ \\
\hline Posterior Mean & 0.030 & -0.001 & 0.089 & 0.054 & 0.142 \\
Posterior Interval & $(0.012,0.051)$ & $(-0.027,0.011)$ & $(0.070,0.109)$ & $(0.053,0.055)$ & $(0.119,0.165)$ \\
MLE & 0.0395 & -0.012 & 0.090 & 0.054 & 0.147 \\
SE & $(0.026)$ & $(0.020)$ & $(0.016)$ & $(0.0007)$ & $(0.017)$ \\
Conf. Interval & $(0,0.082)$ & $(-0.045,0.021)$ & $(0.064,0.116)$ & $(0.053,0.055)$ & $(0.119,0.175)$ \\
\hline
\end{tabular}

Table 3: Estimates of the copula parameters (upper half), and corresponding first order serial dependence metrics (lower half), for the USD/AUD exchange rate series. The mixture copula $c^{M I X}$ with t copula components for $c^{a}$ and $c^{b}$ was used for $c_{2}$. Both Bayesian posterior mean and MLEs are reported. Also reported for the former are $90 \%$ probability intervals, and for the latter, standard errors (SE) and asymptotic 90\% confidence intervals constrained to feasible regions. The metrics include Spearman's rho for dependence in the series $\left(\rho_{1}^{y}\right)$ and volatility $\left(\rho_{1}^{v}\right)$, extremal tail dependence in the series $\left(\lambda_{\text {low }}^{y}=\lambda_{\text {up }}^{y}\right)$, and lower and upper quantile dependence in the volatility at quantile $\alpha=0.05$, $\left(\lambda_{\text {low }}^{v}(0.05)\right.$ and $\left.\lambda_{\text {up }}^{v}(0.05)\right)$.

\begin{tabular}{lccccc}
\hline \multicolumn{7}{c}{ Model } & $\rho_{1}^{y}$ & $\rho_{1}^{v}$ & $\lambda_{\text {low }}^{v}(0.05)$ & $\lambda_{\text {up }}^{v}(0.05)$ & DIC $_{2}$ \\
\hline Copula A1 & -0.001 & 0.089 & 0.054 & 0.147 & -67.446 \\
Copula A5 & -0.001 & 0.074 & 0.053 & 0.125 & -289.892 \\
Copula B1 & -0.012 & 0.079 & 0.054 & 0.148 & -67.359 \\
Copula B5 & 0.003 & 0.066 & 0.053 & 0.127 & -277.477 \\
Copula T1 & -0.009 & 0.088 & 0.054 & 0.143 & -69.280 \\
Copula T5 & 0.000 & 0.071 & 0.054 & 0.124 & -285.537 \\
ARCH(1) & -0.002 & 0.115 & 0.055 & 0.174 & - \\
GARCH(1,1) & 0 & 0.137 & 0.057 & 0.173 & - \\
EGARCH(1,1) & -0.002 & 0.115 & 0.056 & 0.174 & - \\
GARCH-t $(1,1)$ & -0.001 & 0.084 & 0.055 & 0.138 & - \\
\hline
\end{tabular}

Table 4: Dependence metrics for copula and GARCH models fit to the USD/AUD exchange rate returns. These were computed using numerical integration for the first order Copula A1, B1 and T1 models. For all other models, the metrics were computed by simulation. The Deviance Information Criterion $\left(\mathrm{DIC}_{2}\right)$ is reported for the copula models, but not the GARCH models because they are estimated by MLE. 


\begin{tabular}{lcccccc}
\hline & \multicolumn{6}{c}{ Quantile $\alpha$} \\
Model & 1 & 5 & 10 & 90 & 95 & 99 \\
\hline Copula A1 & 0.98 & 4.83 & 9.27 & 90.57 & 95.47 & 99.15 \\
Copula A5 & 1.04 & 4.85 & 9.54 & 90.59 & 95.28 & 99.24 \\
Copula B1 & 0.95 & 4.93 & 9.62 & 90.29 & 95.39 & 99.13 \\
Copula B5 & 1.01 & 5.07 & 9.65 & 90.21 & 95.23 & 99.24 \\
Copula T1 & 0.90 & $4.72^{*}$ & $9.54^{* *}$ & 90.49 & 95.45 & 99.35 \\
Copula T5 & 0.98 & 4.96 & 9.46 & 90.84 & 95.56 & 99.26 \\
ARCH(1) & $1.66^{* *}$ & 4.36 & $7.28^{* *}$ & $92.48^{* *}$ & $96.37^{* *}$ & 99.05 \\
GARCH(1,1) & $1.61^{* *}$ & 5.07 & $9.11^{*}$ & $91.63^{* *}$ & $95.86^{*}$ & 99.18 \\
\hline EGARCH(1,1) & $1.69^{* *}$ & 4.88 & 9.43 & $91.38^{* *}$ & $95.64^{*}$ & 99.18 \\
GARCH-t $(1,1)$ & 1.31 & 5.62 & 10.22 & $90.13^{*}$ & 95.39 & $99.37^{*}$ \\
\hline
\end{tabular}

Table 5: Mean exceedances $\hat{\alpha}$ (in percent) over $T-1=3668$ days of one day ahead VaR forecasts of USD/AUD exchange rate returns. Results are given for ten models and six different quantile values. Rejection of the null hypothesis of the conditional coverage Christoffersen (1998) test is denoted with '*' and '**' at the $95 \%$ and $99 \%$ level of confidence, respectively.

\begin{tabular}{ccccc}
\hline & \multicolumn{5}{c}{ Fitted Model/Correct Model } \\
\cline { 2 - 5 } $\begin{array}{c}\text { Volatility serial } \\
\text { dependence }\end{array}$ & $\begin{array}{c}\text { Copula B1/ } \\
\text { ARCH(1) }\end{array}$ & ARCH(1)/ & Copula B5/ & GARCH(1,1)/ \\
Copula B1 & GARCH(1,1) & Copula B5 \\
\hline$\rho_{1}^{v}$ & 1.16 & 3.41 & 1.04 & 3.25 \\
$\rho_{2}^{v}$ & 0.99 & 4.77 & 1.10 & 1.22 \\
$\rho_{3}^{v}$ & 0.89 & 6.64 & 1.07 & 1.64 \\
$\rho_{4}^{v}$ & 0.79 & 5.52 & 1.08 & 2.36 \\
$\rho_{5}^{v}$ & 0.98 & 2.06 & 1.06 & 2.32 \\
\hline
\end{tabular}

Table 6: Relative RMSE of the estimates of $\rho_{1}^{v}, \ldots, \rho_{5}^{v}$ of the fitted model over the correct model. Four mis-specified models are considered. 


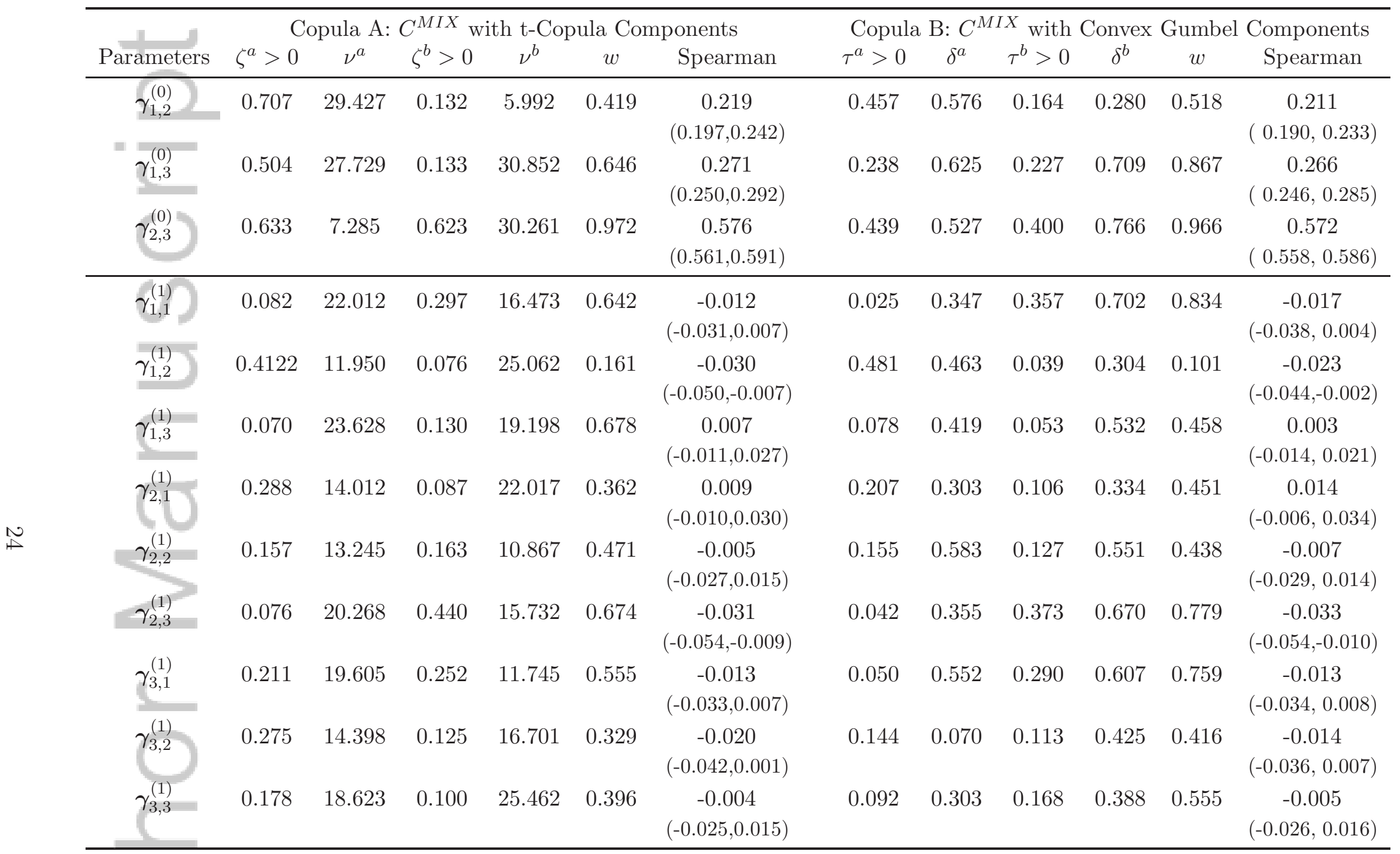

Table 7: Posterior means of the pair-copula parameters for the D-vines fit to the three-dimensional exchange rate return series. The lefthand side gives the pair-copula parameters for Copula A, and the righthand side for Copula B. The posterior mean and $90 \%$ probability intervals are also given for the Spearman's rho of each pair copula. The USD/JPY, USD/AUD and USD/EUR returns are denoted as series 1, 2 and 3, respectively.

This article is protected by copyright. All rights reserved. 


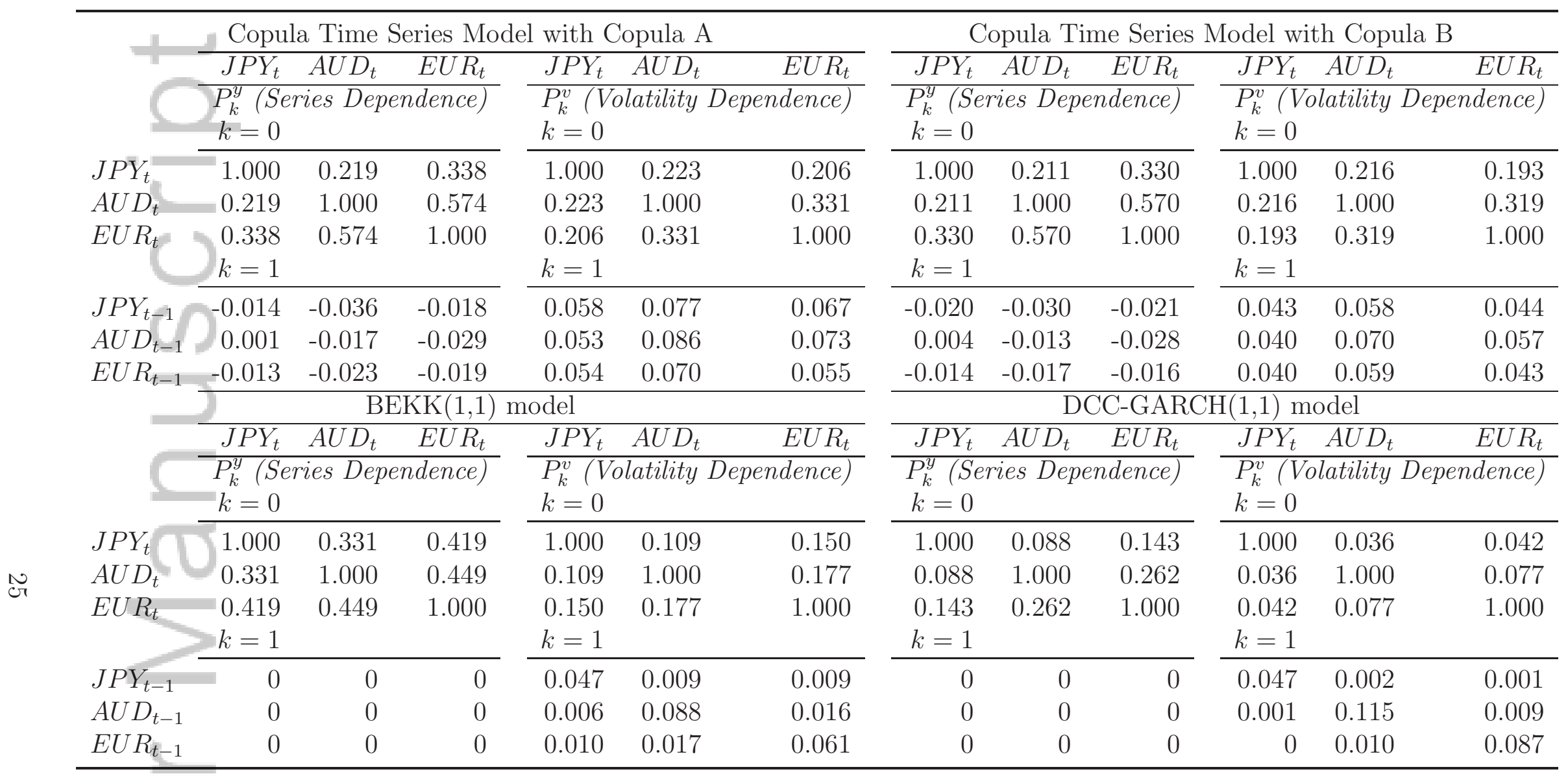

Table 8: Matrices of pairwise Spearman correlations in the series $\left(P_{k}^{y}\right.$; left-hand side) and the volatility $\left(P_{k}^{v}\right.$; right-hand side) for the three-dimensional exchange rate example. Results are reported separately for the two fitted copula models, and the BEKK and DCC models. The daily returns on the USD/JPY, USD/AUD and USD/EUR exchange rates are denoted at time $t$ as $J P Y_{t}, A U D_{t}$ and $E U R_{t}$, respectively. For each model, the top rows give cross-sectional dependence $(k=0)$, and the bottom rows give first order serial dependence $(k=1)$. For example, for the time series copula model with Copula A, the pairwise Spearman correlation between $J P Y_{t-1}$ and $E U R_{t}$ is $\rho_{J P Y, E U R, 1}^{y}=-0.018$, and the corresponding volatility spillover is $\rho_{J P Y, E U R, 1}^{v}=0.067$.

This article is protected by copyright. All rights reserved. 


\begin{tabular}{lcccccc}
\hline & \multicolumn{7}{c}{ Quantile $\alpha$} \\
Model & 1 & 5 & 10 & 90 & 95 & 99 \\
\hline Copula A & 0.82 & 4.47 & 9.54 & 89.56 & 95.12 & 99.32 \\
Copula B & 0.65 & 4.36 & 9.51 & 89.50 & 94.85 & 99.24 \\
BEKK(1,0) & $1.50^{*}$ & 4.63 & $8.15^{* *}$ & $90.95^{*}$ & 95.26 & 98.77 \\
BEKK $(1,1)$ & 1.28 & 4.63 & $8.37^{* *}$ & $90.51^{*}$ & 94.98 & 98.96 \\
DCC-GARCH $(1,1)$ & 1.34 & 4.77 & $8.51^{* *}$ & $90.27^{*}$ & 94.93 & 98.88 \\
\hline
\end{tabular}

Table 9: Mean exceedances $\hat{\alpha}$ (in percent) over $T-1=3668$ days of one day ahead VaR forecasts of returns on an equally-weighted currency portfolio. Results are given for five multivariate models and six different quantile values. Rejection of the null hypothesis of the conditional coverage Christoffersen (1998) test is denoted with ' ${ }^{*}$ ' and ' $* *$ ' at the $95 \%$ and $99 \%$ level of confidence, respectively.
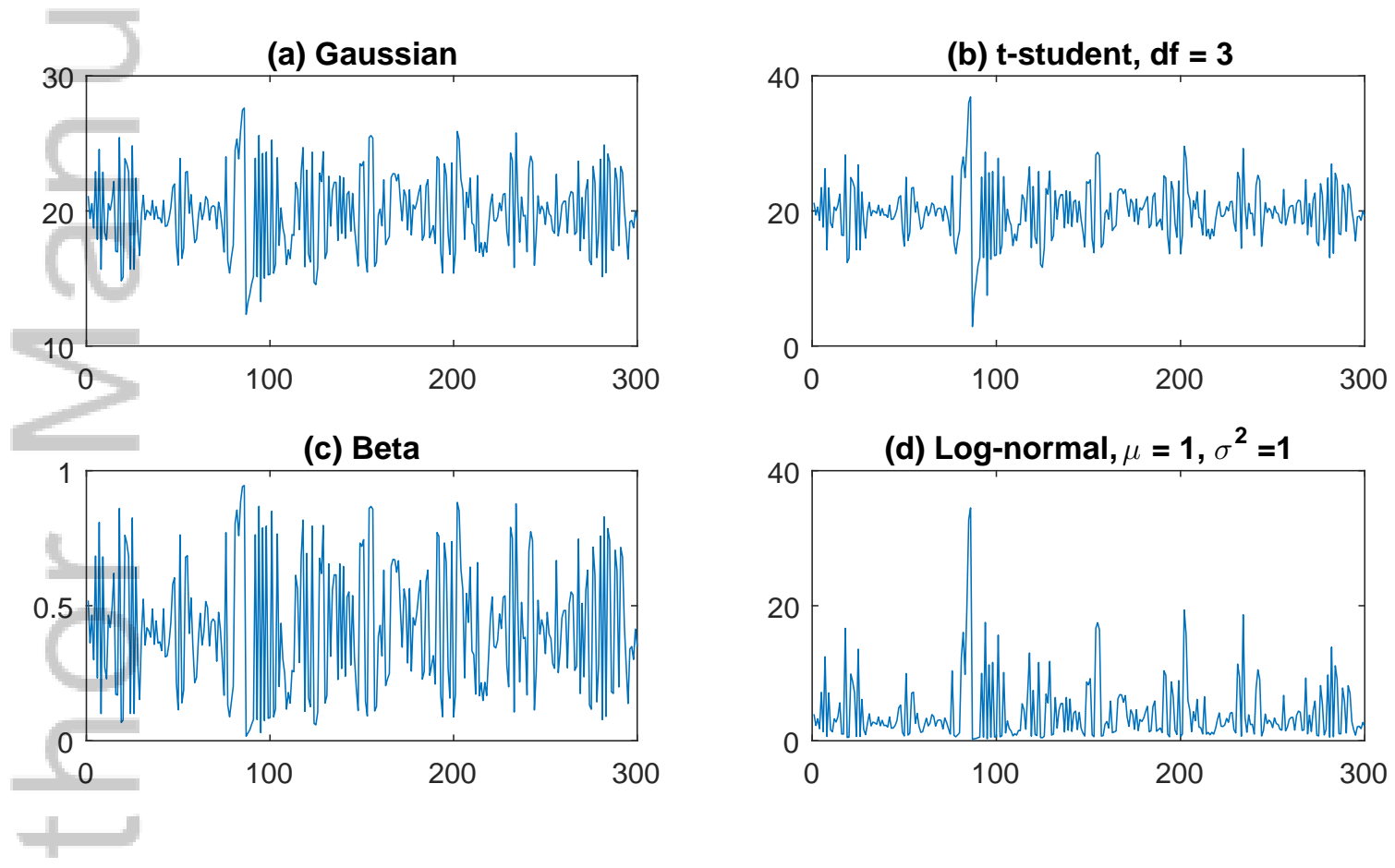

Figure 1: Four heteroskedastic series simulated from the time series copula model. A vector $\boldsymbol{u}$ of length $T=300$ is simulated from the copula at Equation (3) with $c_{2}=c^{M I X}$, where $w=0.5$, and $c^{a}, c^{b}$ are identical t copula densities with parameters $(\zeta=0.9, \nu=3)$. We then compute $y_{t}=F^{-1}\left(u_{t}\right)$ using the quantile function $F^{-1}$ of the four time invariant marginal distributions: (a) $Y_{t} \sim N(20,3)$, (b) $Y_{t} \sim 20+3 t_{3}$, (c) $Y_{t} \sim \operatorname{Beta}(1.5,2)$ and (d) $\ln \left(Y_{t}\right) \sim N(1,1)$. 
(a)
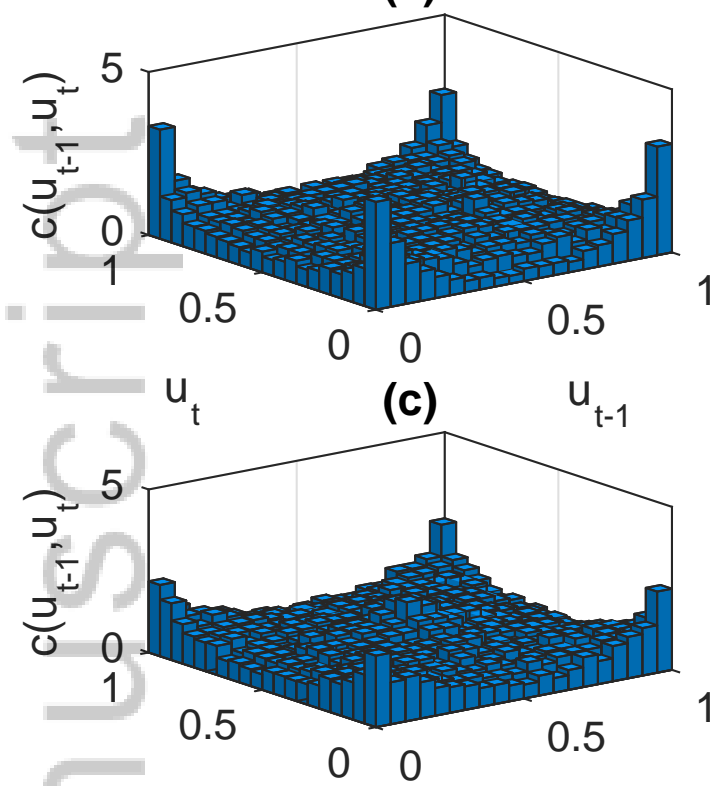

(e) $\quad u_{t-1}$

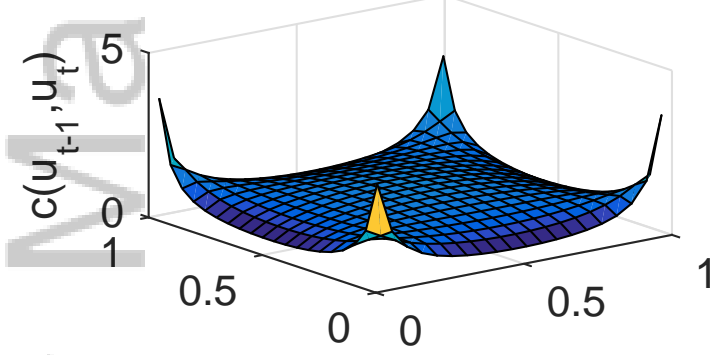

(g) $\quad u_{t-1}$

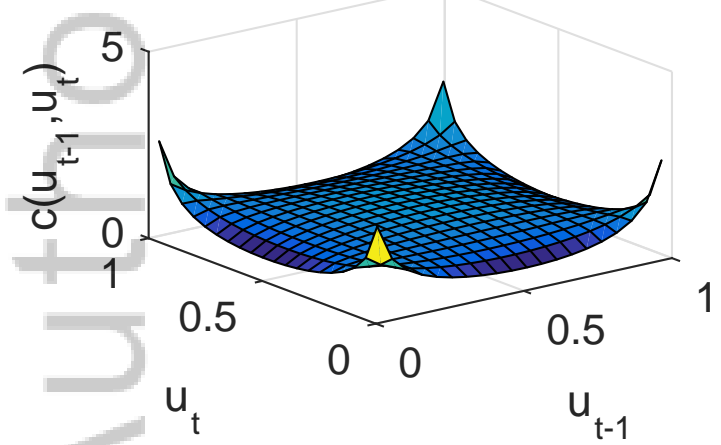

(b)
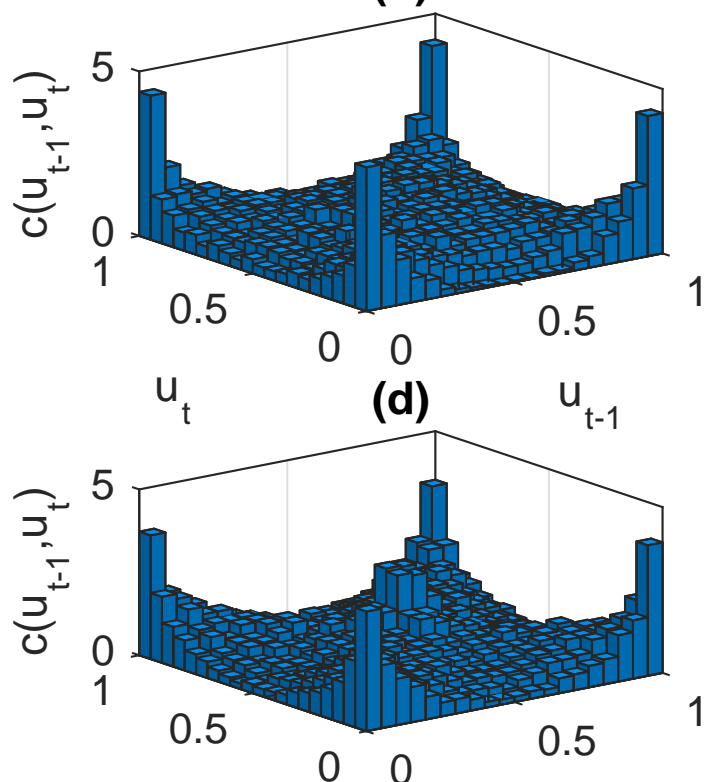

(f)

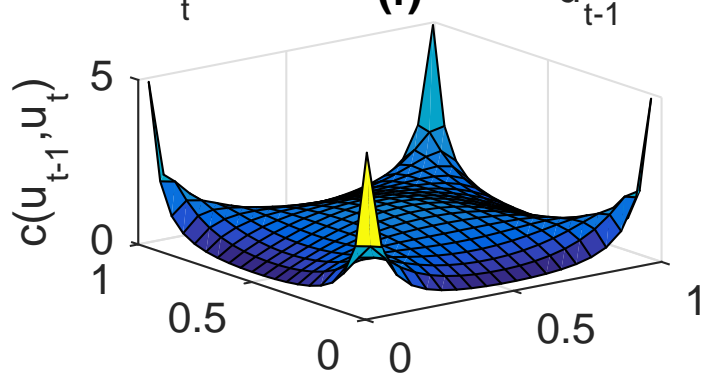

(h) $u_{t-1}$

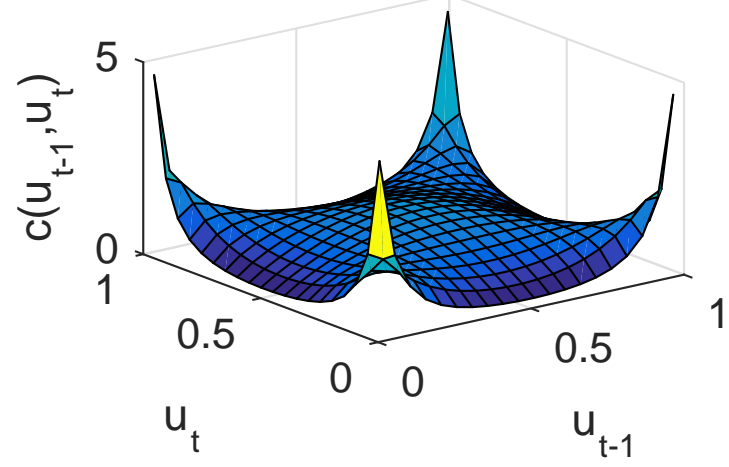

Figure 2: Copula densities $c_{2}$ for heteroskedastic time series. Panels (a) and (b) present the empirical copula densities for the $\operatorname{ARCH}(1)$ processes with $\alpha_{1}=0.5$ and $\alpha_{1}=0.9$, respectively. Panels (c) and (d) display the empirical density copulas for the SV(1) processes with $\phi_{1}=0.5$ and $\phi_{1}=0.9$, respectively. Panels (e) to (h) plot the mixture copulas $c^{M I X}$ (with t-copula components) fitted to the same data used to compute the densities in panels (a) to (d), respectively. 
(a)

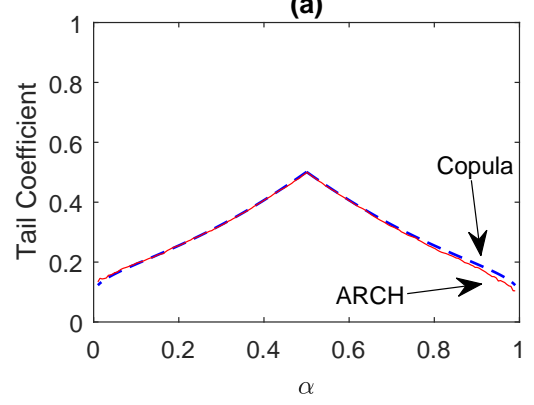

(c)

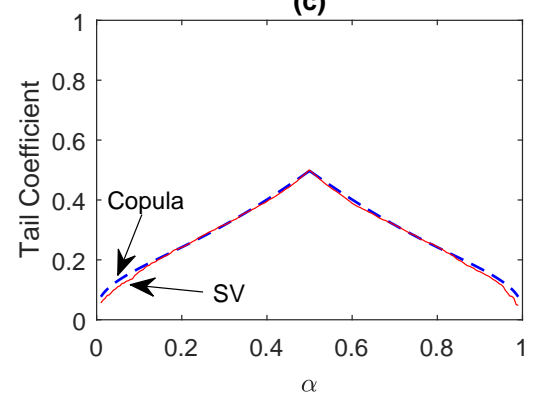

(b)

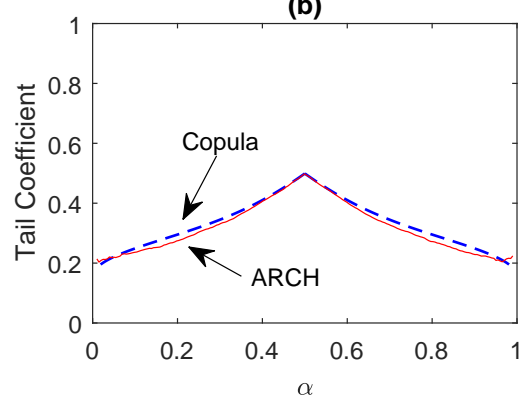

(d)

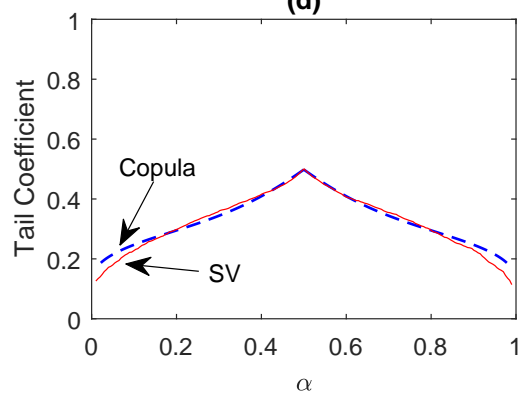

Figure 3: Quantile dependence coefficients for $\mathrm{ARCH}(1)$ and $\mathrm{SV}(1)$ models. Panels (a) and (b) display the quantile coefficients for the $\mathrm{ARCH}(1)$ processes with $\alpha_{1}=0.5$ and $\alpha_{1}=0.9$, respectively. Panels (c) and (d) show the quantile coefficients for the SV(1) processes with $\phi_{1}=0.5$ and $\phi_{1}=0.9$. The coefficient $\lambda_{\text {low }}(\alpha)$ is plotted for $\alpha<0.5$, and $\lambda_{\text {up }}(\alpha)$ for $\alpha>0.5$. The solid red line gives the empirical quantile coefficients, and the dashed blue line that from the copula model.

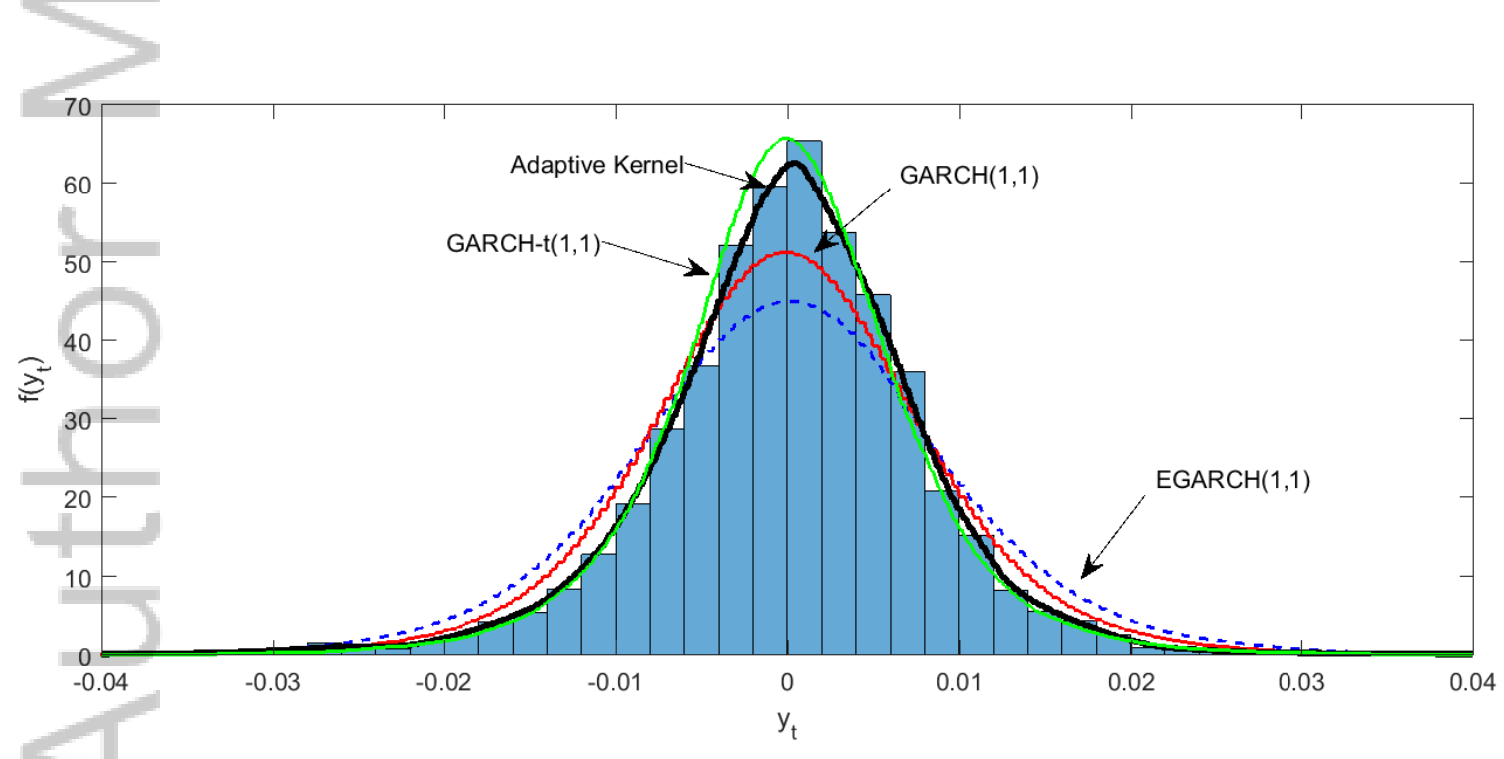

Figure 4: Histogram of daily USD/AUD returns, the locally adaptive kernel density estimate (thick black line), and the marginal distributions of fitted $\operatorname{EGARCH}(1,1)$ (blue dashed line), $\operatorname{GARCH}(1,1)$ (red line) and GARCH-t(1,1) (green line) models. 
(a)

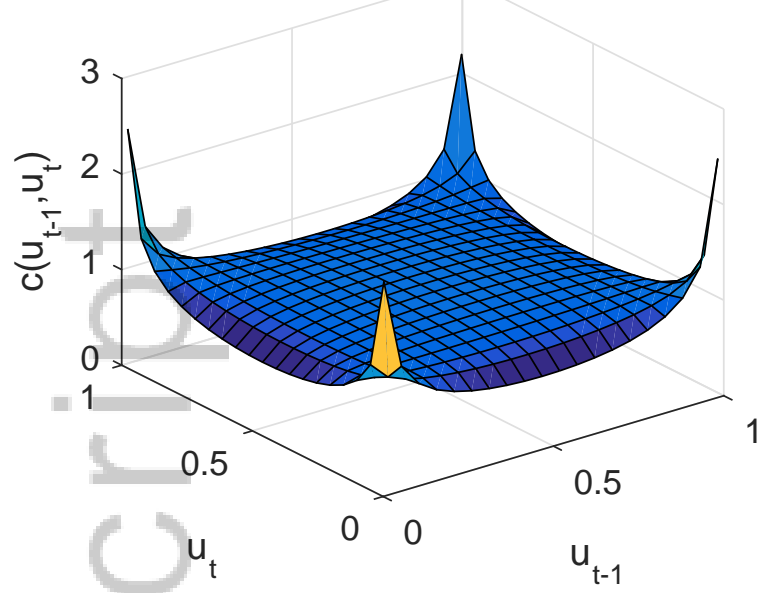

(b)

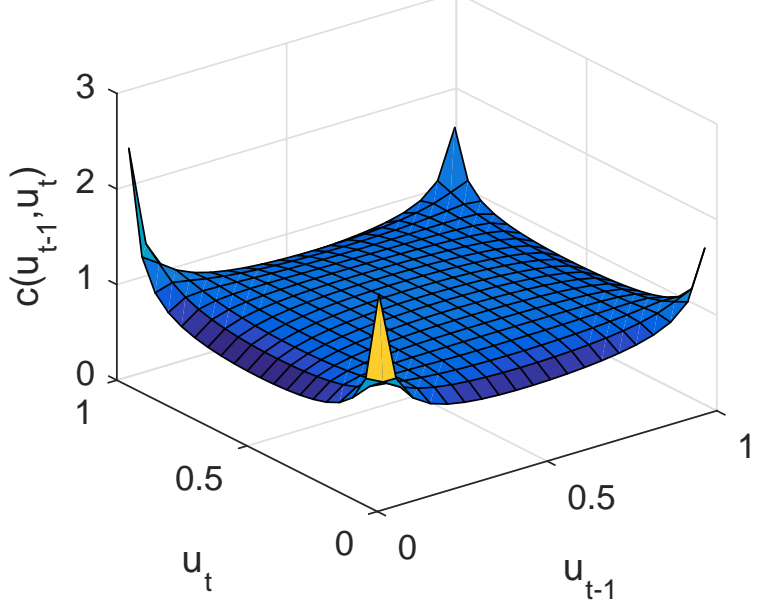

Figure 5: Bivariate copula densities $c_{2}$ fitted to the USD/AUD returns. A mixture copula was used with t-copula components in panel (a), and convex Gumbel components in panel (b).
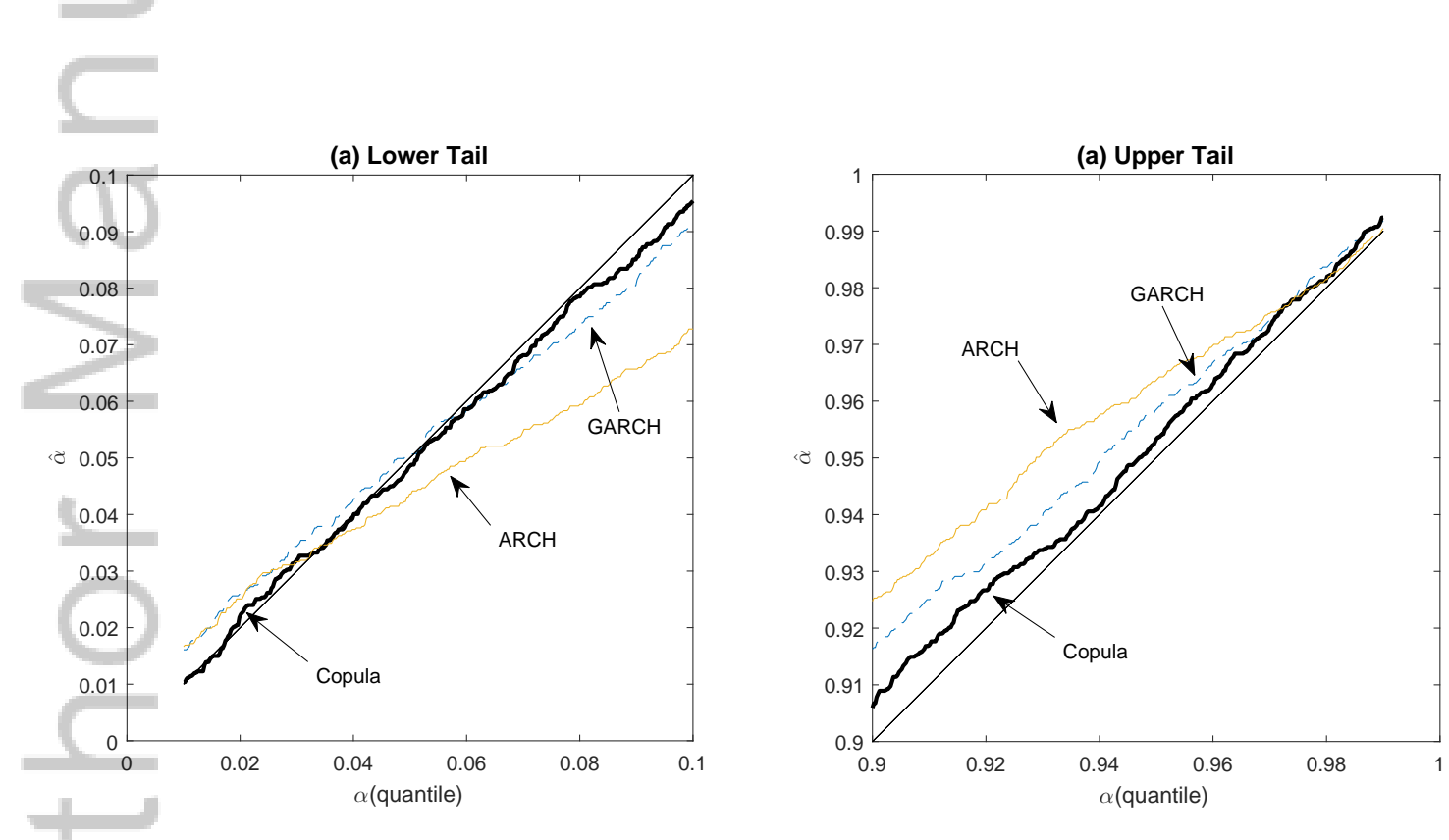

Figure 6: Coverage results for one day ahead VaR predictions for USD/AUD daily returns. Panel (a) displays results for the lower tail, and panel (b) for the upper tail. Results are given for the ARCH(1), GARCH $(1,1)$ and Copula A5 models. See Figure ?? of the Online Appendix for an expanded colour figure that also includes results for the $\operatorname{EGARCH}(1,1)$ and $\operatorname{GARCH}-\mathrm{t}(1,1)$ models. 
(a) JPY

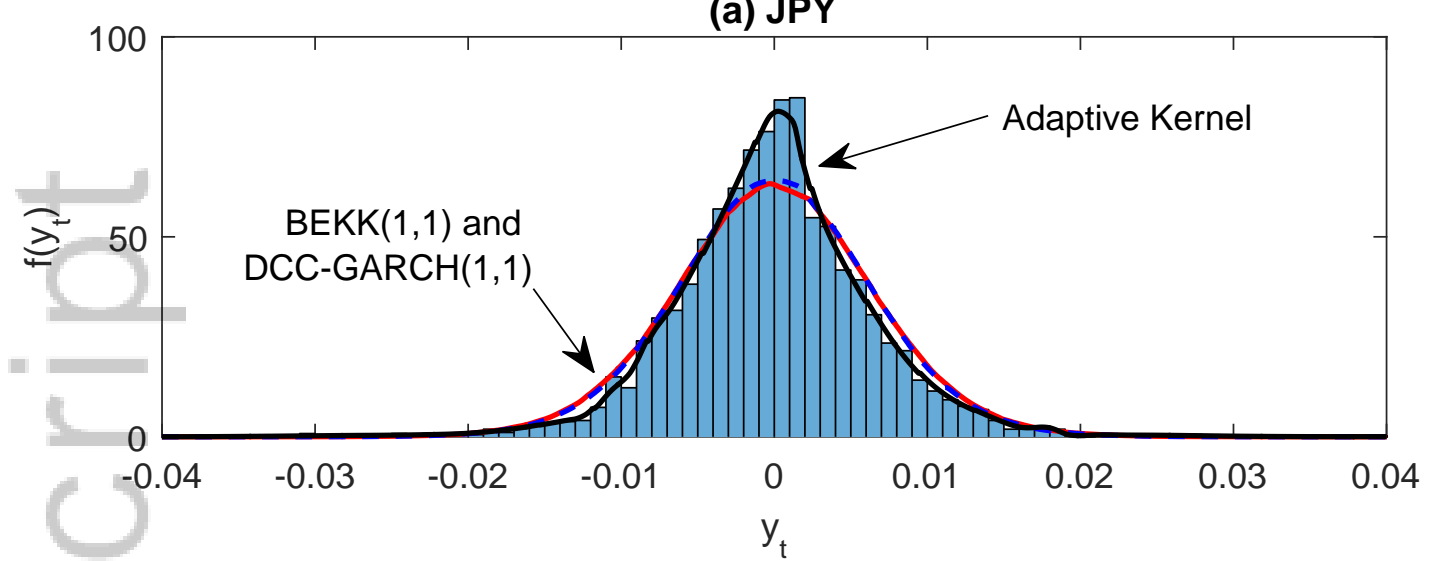

(b) AUD

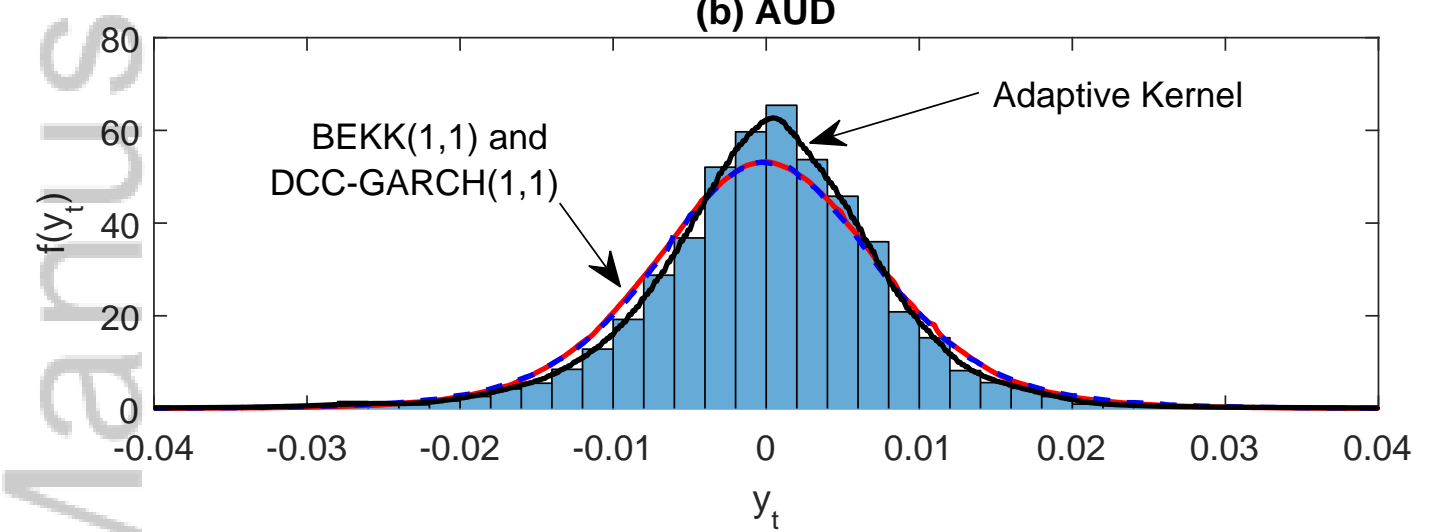

(c) EURO

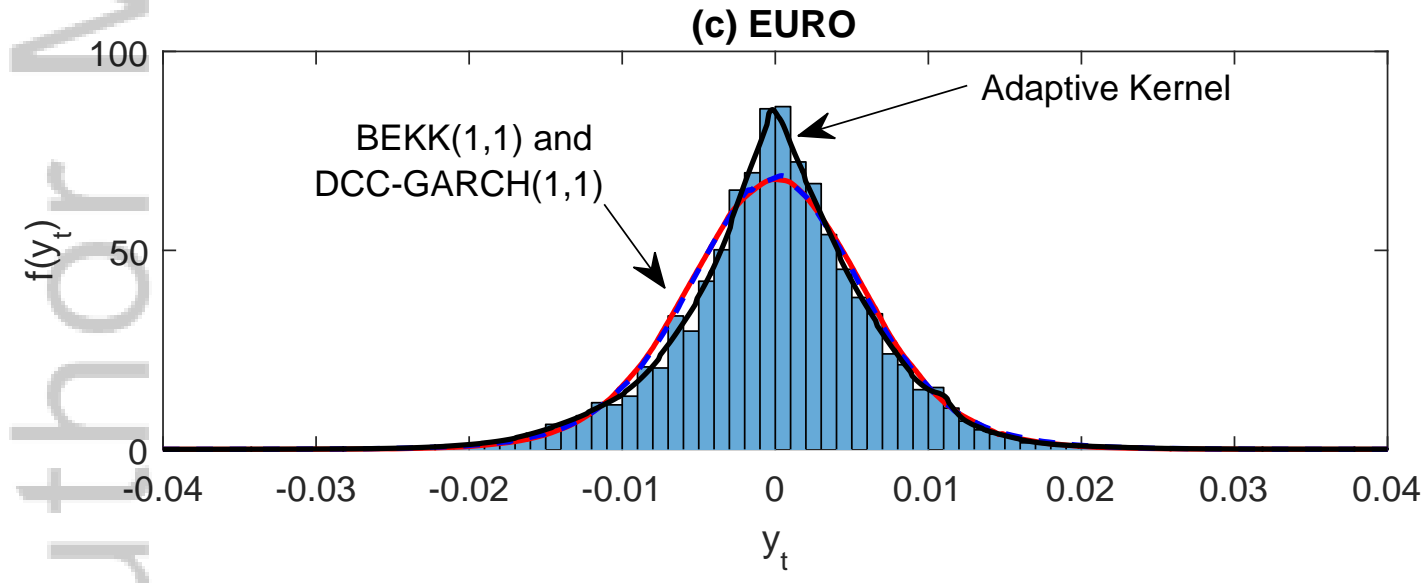

Figure 7: Histograms of daily foreign exchange returns on (a) USD/JPY, (b) USD/AUD and (c) USD/EUR. Also plotted are the locally adaptive kernel density estimates (black lines), and the marginal distributions of the fitted $\operatorname{BEKK}(1,1)$ (red lines) and DCC-GARCH $(1,1)$ (blue dashed lines) models. 

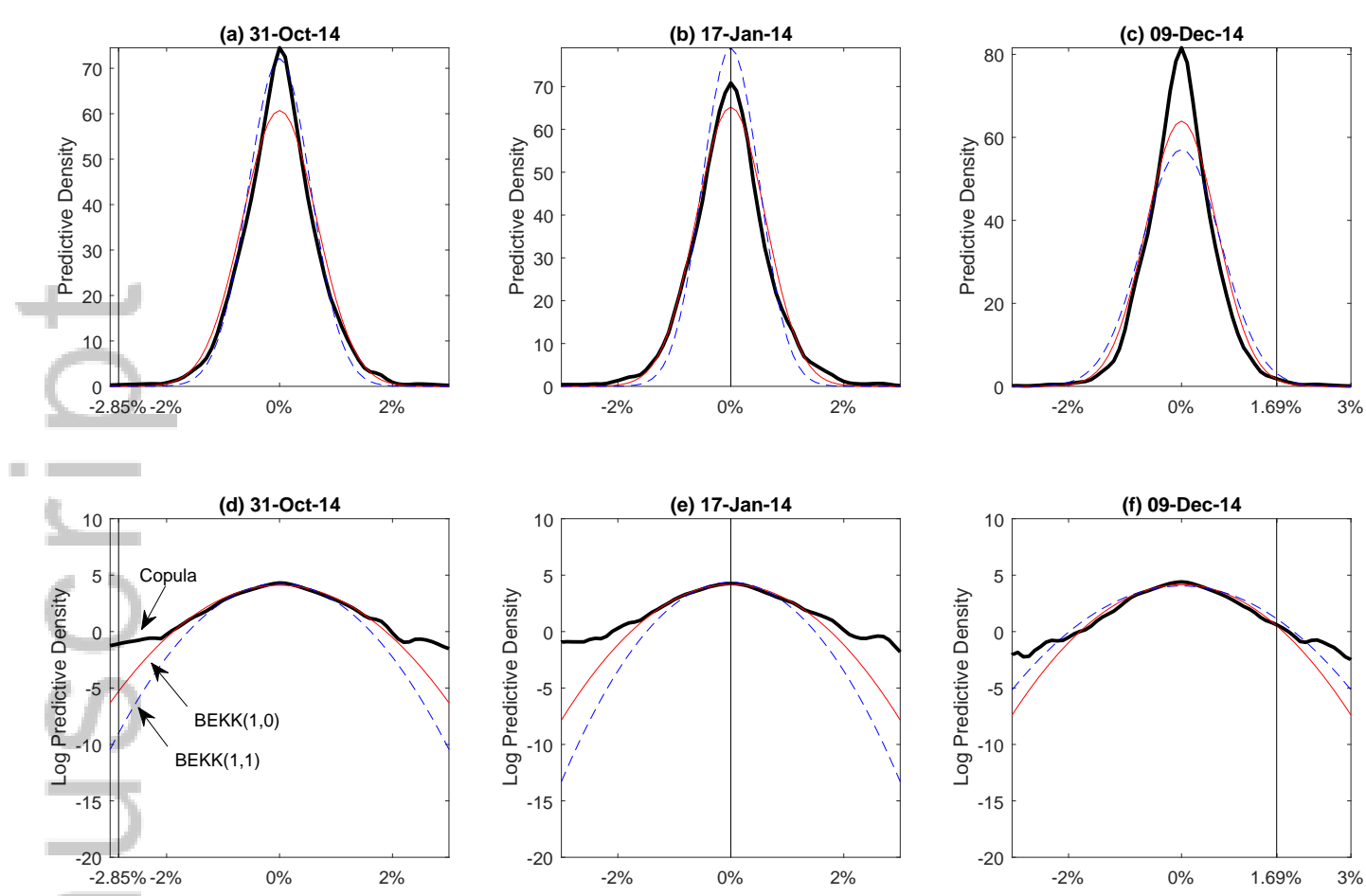

Figure 8: Panels $(\mathrm{a}-\mathrm{c})$ provide one day ahead forecast densities for the USD/JPY return from the Copula A (thick black line), $\operatorname{BEKK}(1,0)$ (red line) and $\operatorname{BEKK}(1,1)$ models (blue dashed line), respectively. Panels $(\mathrm{d}-\mathrm{e})$ plot the logarithm of the same three forecast densities, to aid comparison of the tails. Forecasts are made for three specific days: (a,d) 31 Oct. 2014; (b,e) 17 Jan. 2014; and, (c,f) 9 Dec. 2014. These days have the smallest, largest and 'most close to zero' returns observed during the last 18 months of the series. Vertical lines plot these observed values. 


\section{University Library}

\section{- M M N E R VA A gateway to Melbourne's research publications}

Minerva Access is the Institutional Repository of The University of Melbourne

Author/s:

Loaiza-Maya, R;Smith, MS;Maneesoonthorn, W

Title:

Time series copulas for heteroskedastic data

Date:

2018-04-01

Citation:

Loaiza-Maya, R., Smith, M. S. \& Maneesoonthorn, W. (2018). Time series copulas for heteroskedastic data. JOURNAL OF APPLIED ECONOMETRICS, 33 (3), pp.332-354. https:// doi.org/10.1002/jae.2610.

Persistent Link:

http://hdl.handle.net/11343/293831 